\title{
Robotic Ad-hoc Networks Connectivity Maintenance based on RF Signal Strength Mapping
}

\author{
Mustafa Ayad ${ }^{1}$, Mohamed Ayad ${ }^{3}$ \\ Electrical and Computer Engineering \\ The State University of New York at Oswego \\ Oswego, NY, USA
}

\author{
Richard Voyles ${ }^{2}$ \\ Electrical and Computer Engineering Technology \\ Purdue University at West Lafayette \\ West Lafayette, IN, USA
}

\begin{abstract}
Network connectivity preservation is one of the substantial factors in achieving efficient mobile robot teams' maneuverability. We present a connectivity maintenance method for a robot team's communication. The proposed approach augments the Radio Frequency Mapping Recognition (RFMR) method and the signal strength gradient decent approach for an overall goal to create a Proactive Motion Control Algorithm (PMCA). The PMCA algorithm controls and helps strengthen mobile communicating robots' connectivity in the existent Radio Frequency (RF) obstacles. The RFMR method takes advantage of Hidden Markov Models (HMMs) results, which assist in learning electromagnetic environments depending on measurements of RF signal strength. The classification results of HMM lead the robots to resolve whether to continue the current trajectory for avoiding the obstacle shadow or move back to desirable robust Signal Strength (SS) positions. In both cases, the robot will run the gradient approach to determine the signal change trend and drive the robot toward the strong SS direction for maintaining link connectivity. The PMCA, depending on the results of RFMR and gradient approaches, promises to preserve robots' motion control and link connectivity maintenance.
\end{abstract}

Keywords-RF mapping recognition; link connectivity; gradient algorithm

\section{INTRODUCTION}

The majority of Communication networks, especially wireless networks, are deployed in territories with different interference sources (Different obstacles), affecting the communication signals and creating no Line Of Sight (LOS) among communication devices, so they can not identify each other. However, the Frezonet zone where the signal propagates should be free of interferences sources such as conducting and conducting obstacles of different types to an actual LOS $[1,2]$. One problem of the RF communications in disasters such as crumpled buildings is many signal interference sources that cause no LOS and disrupt the communication signal. Robot swarms of small size can collaborate in search and rescue environments and accomplish tasks that no one robot can complete alone [3]. Fig. 1 illustrates the urban search and rescue (USAR) robot team collaborating and communicating to transmit data to the network base station (BS). The robot team will encounter many problems when discovering the collapsed area. One of the critical problems is maintaining a reliable link between the robot team members to transmit the message to the BS. For example, a single robot could not send messages directly from the most distant network topology to the BS. What's more, each robot in the team has different duties. For example, it searches for survivors, maintains communication through network topology, and transmits data to the BS.

Collaborating teams of small robots can facilitate tasks beneficial to monitoring, surveillance, and other rescue services in unsafe locations [4]. However, they have limited mobility, power, and communication coverage [5]. Consequently, the system resources are distributed among multiple robots, which work as a team to accomplish a mission. Hence, each small robot in the collaborating team has inadequate sensing and processing abilities for the assigned tasks, e.g., mapping the collapsed area, transmitting acquired data to BS, and carrying necessary sensors for the mission.

A robot in the robotic network can quickly lose communication with team members while collaborating. Therefore reliable strategies for wireless communication are essential [6]. Consequently, a dedicated link maintenance strategy is vital for reliable mobile ad hoc networks (MANETs) connectivity, particularly when the network experiences sporadic connectivity caused by hostile environments. Hence, network connectivity maintenance is a target for achieving adequate network performance. In this context, it is possible to employ the variations in the SS measurements in control algorithms that control motion and preserve connectivity.

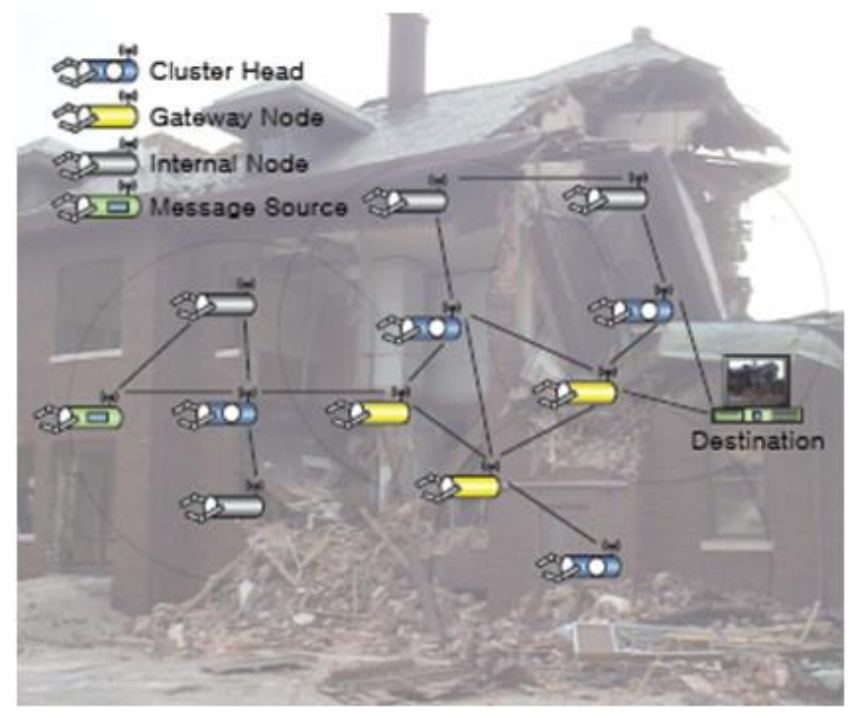

Fig. 1. Robots Warm in a Collapsed Building. 
Furthermore, significant developments in robotic networks have led to reliable, self-organizing communication schemes that do not require the collaboration of robots with existing communication infrastructure. Besides, the communication scheme has given bonds to the motion control concept for movable agents, tied to the robot's movement ability to reach proper positions in the field to preserve connectivity and accomplish assigned tasks [7,8]. In [9], the authors manifest the possibility of designing trajectories by co-optimizing sensing and communication information when motion planning.

This article introduces the RFMR method, which uses SS measurements collected from the field to discover, identify, and identify hostile environments with different RF obstacles. In addition, this method also studies the impact of RF obstacles on RF-SS in various scenarios. In addition, according to the RFMR method, we propose a SS gradient algorithm to control the movement trend of the robot. Most up-to-date gradient strategies use a standing interference source to compute a two-dimensional (2-D) gradient to an appointed stable source of the signal source, similar to regression in a 2-D space. However, these approaches did not extend their gradient calculation to nodes in motion that require a four-dimensional (4-D) space gradient estimation. Consequently, augmenting the gradient algorithm and RFMR approach will support creating the PMCA control algorithm to preserve mobile robots' successful communication in the appearance of RF obstacles.

In the simulation and physical experiments, two robots are moved in a different direction around RF obstacles and study their effect on the measurements of the RF signal. The interference sources include cages, walls, and cylinders of various dimensions. The obstacles used are conductively made of a Perfect Electrical Conductor (PEC). When robots move and collect SS measurements around a fixed position obstacle, the collected SS string along the robot's path retains useful information for obstacle recognition and classification. First, the collected SS measurements were segmented, and then features (observation sequence) were extracted using Fast Fourier Transform FFT [4,5]. Afterward, the observation sequences are coded using a clustering algorithm known as $\mathrm{K}$ means [10]. Then, first-order HMMs are used to model the observation sequences $[11,12]$, trained, and then used for the RFMR method. Using this approach, the outcomes of the experiments show very accurate recognition results. As the movable robots identify the nature and assess the dimensions of the confronted obstacle, the PMCA will decide whether to continue moving along the current trajectory to bypass the RF obstacle's shadow or reverse drive to a position where the robot gains a reliable SS. In either case, the gradient descent algorithm is applied, and the multi-dimensional gradient of the strong SS direction used by the robot PMCA for connection maintenance is estimated.

In short, our proposed robot PMCA for preserving communication links and fixing disrupted links is implemented depending on RFMR and gradient methods. The RFMR method uses HMM to discover the RF environment based on SS measurements to estimate the type and size of obstacles. The gradient algorithm outcome decides the direction of the robust SS to maintain connectivity. Finally, the robot motion control can keep the connection and repair the broken link depending on the RFMR and gradient results. The PMCA algorithm's reliability and performance were tested by conducting various simulation experiments. Consequently, the proposed approach has exhibited assuring solutions for the connectivity problem of a robotic network.

We organized this paper as follows. The relevant prior work in a controlled mobile sensor network, estimating and mapping radio signals, is briefly introduced in Section II. Section III presents the RFMR method formulation and modeling to justify this new development. In Section IV, the physical obstacle experiments are described. The simulation and physical results validation is described in Section V. Section VI explains the obstacle parameterization. In Sections VII and VIII, RFMR based on HMM and numerical results are explained. PMCA and gradient methods are described in Sections IX and $\mathrm{X}$. The experimental gradient results are presented in Section XI. The control motion algorithm simulation is illustrated in XII. Section XIII presents conclusions and future work.

\section{LITERATURE AND RELATED WORK}

Recently, connectivity and SS measurements have become essential attributes of communication networks to ensure quality communication $[13,14]$. In addition, the robot network should maintain connectivity when performing tasks [15]. Based on the information from radio SS, authors in [16] calculate the 2-D gradient of a robot in motion. Besides, the authors calculated the gradient of the robot in mobility to a stationary source of RF signal. In [17], the possibility of localizing and navigating to a standstill source of RF signal by utilizing the two-dimensional gradient of a cooperating sensor network is studied. Authors [15,17] defined a 2-D gradient for a robot in motion to a standstill source of RF signal. The robot follows a predefined trajectory to accommodate its velocity. Authors in [18] proposed a probabilistic framework for evaluating wireless channels. Authors in [19] developed tools for estimating and mapping radio signals. In an attempt to create an urban radio map, Authors in [20] constructed a BS in an unknown location, which transmits data to one or more mobile robots to create a map of the radio signal for a specified area. An algorithm that sets the team's goals and controls its movement makes sure it reaches designated targets without degrading the quality of the link maintaining the map.

Moreover, [20] discussed experimental validation of a procedure that automatically conserves the connection between collaborating robots over such a distributed network. A feedback control framework that is distributed and does not impose restrictions on the network's structure except for desired connectivity specifications has been proposed by [21] concerning the local connectivity of a network. In [22], the authors introduce a measure that provides a measure of the network's global connectivity if certain conditions are met. The authors [22] solved stratum stability's distributed maintenance problem with the nearest neighbor links. Authors propose robots to overcome environmental interference and enable end-to-end communication [23,24]. Several measurements in the robot network are used to estimate the 
spatial variation of the wireless channel by [25], where the link quality predicts communication.

Current research on wireless sensor networks focuses on developing energy-saving routing protocols, distributed data compression, transmission schemes, and cooperative signal processing algorithms [26]. In addition, our research is interested in creating a wireless video sensor network of robots that work in hazardous areas and accomplish different tasks while maintaining team connectivity. The wireless network of video sensors is a locally distributed mobile sensor system that captures, processes, and transmits information through a self-organizing wireless network, as shown in Fig. 2. Compared with traditional communication systems, wireless video sensor networks operate underneath a unique set of resource restrictions, including airborne computing and transmission bandwidth. In [12], the authors investigated the resource utilization behavior and analyzed the Video sensor network performance under resource constraints.

\section{RFMR FORMULATION AND MODELING}

The RFMR Method depends on the RF-SS determinations on the robot's path. First, the technique identifies and classifies the types of RF shadows on the robot's path. Then, it provides the learned knowledge to PMCA. The outcome of the HMM gained from the RFMR method advises the moving robots underneath the obstacle's special effects. After that PMCA relies on HMM results to determine the proper control on the robot motion, firstly, to recover from the shadow of RF obstacles and then preserve the connectivity of the robot. PMCA decided to let the robot move forward on the current trajectory under the influence of the shadow of the RF obstacle. It did that depending on the size and type of the obstacle. In contrast, PMCA guided them to back movement to a vital SS location and then applied the SS gradient algorithm to find the trend of another robot to communicate.

All RFMR experiments use two mobile sensors transmitting and receiving RF signals at a frequency of 2.4 GHz. They measure the RF-SS at their present location. Multipath, fading, and interference may affect the measured $\mathrm{RF}$ signal [3,29]. Mobile sensors, at $\mathrm{t}=0$, are positioned at $\left(\mathrm{x}_{\mathrm{t}}\right.$, $\mathrm{y}_{\mathrm{t}}$ ) in the 2-D Cartesian space. The mobile sensors 2-D configuration spaces are divided into grids of equal area. The grid width is $\Delta x=L x / M$, where Lx is the length and $\mathrm{M}$ is the number of segments, lengths, and segments along the $\mathrm{x}$-axis. The grid length is $\Delta y=L y / N$, where Ly and $N$ are the lengths and the number of segments in the y-axis. For example, the grid's width might be $\frac{1}{3} \lambda, \frac{2}{3} \lambda$ or $\lambda$, which is $12.5 \mathrm{~cm}$ at 2.4 $\mathrm{GHz}$.

In RFMR simulation experiments, the robots move predefined trajectories to acquire RF-SS measurement. The robot's trajectory, $l$ th, can be expressed by.

$x_{t, l}^{(i)}=x_{0, l}^{(i)}, y_{t, l}^{(i)}=y_{0, l}^{(i)}+k \Delta_{y}, t 1,2, \ldots, N$,

Where the trajectory index is $l$, the robots index is $i \varepsilon\{1,2\}$, the $i$ th robot start location at time $t=0$ is $\left(x_{0 . l}^{(i)}, y_{0 . l}^{(i)}\right)$. The $i$ th robot's motion starts at the initial location $\left(x_{0, l}^{(i)}, y_{0, l}^{(i)}\right)$ and increased by a step size of $\boldsymbol{\Delta}_{\boldsymbol{y}}$ along with the $y$-direction is defined in Equation (1). Besides, the first robot started at $\left(x_{0, l}^{(1)}=l \Delta_{x}, y_{0, l}^{(1)}=0\right)$ for the trajectory, $\boldsymbol{l t h}$. Then, the second robot location is expressed in $x_{0, l}^{(2)}=x_{0, l}^{(1)}+$ $d$, and $y_{0, l}^{(2)}=, y_{0, l}^{(1)}$. The robots have the exact coordinates in the y-axis, and they are a d distance in the x-axis. Fig. 3(a) shows an experiment scenario of two robots. The SS at the receiver antenna can be expressed as.

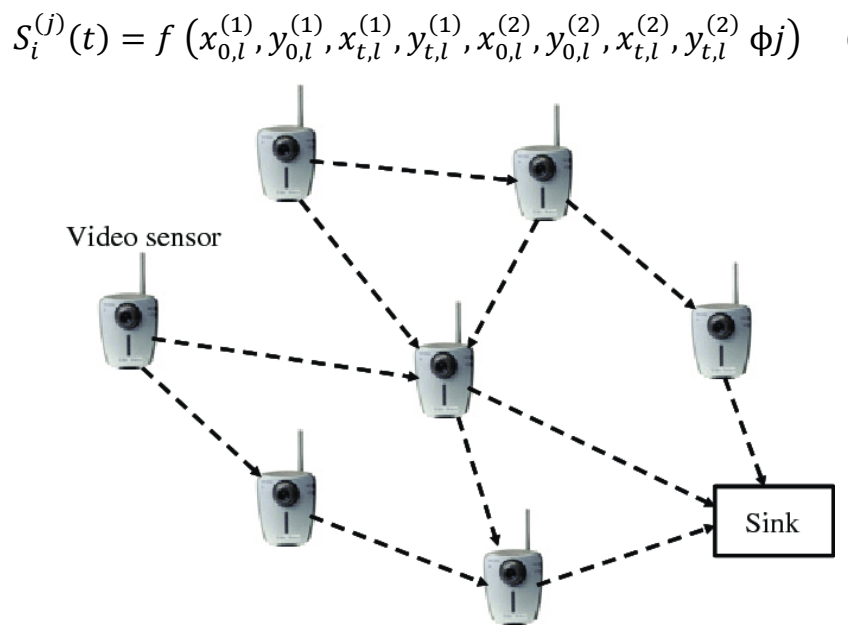

Fig. 2. Wireless Video Sensor Network.

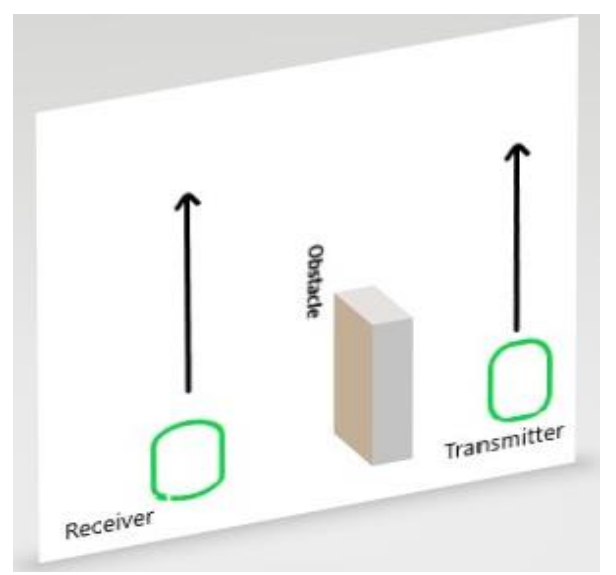

(a)

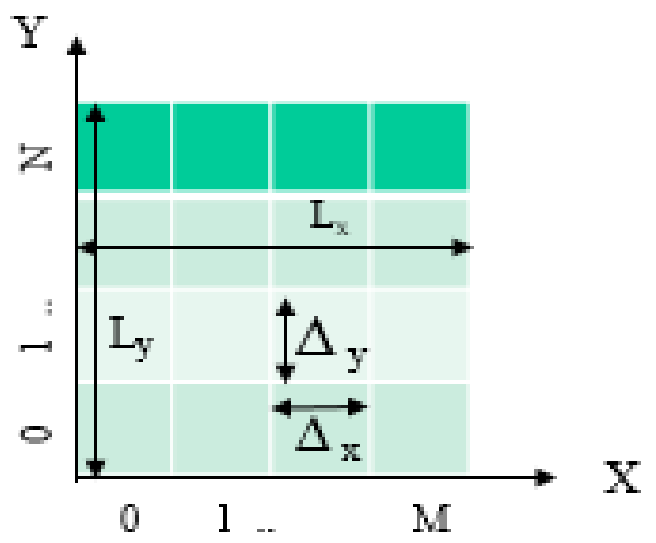

(b)

Fig. 3. (a) A Transmitter and Receiver Exchange about an Obstacle (b) The 2-D configuration Space. 
This is the SS measurement on the trajectory, $l$ th, in the RF obstacle of type $\boldsymbol{j}$ existence at time $t$. The $S_{i}^{(j)}(t)$ function represents the robot's start location $\left(x_{0, l}^{(i)}, y_{0, l}^{(i)}\right)$, time t robot's location $\left(x_{t}^{(i)}, y_{t}^{(i)}\right)$ and the special characteristics of the obstacle $\phi_{j}$. The index of trajectory $l$ is $l=1, \ldots, L^{(j)}$ for each obstacle of type $j . \mathrm{L}^{(j)}$ is the trajectory's number in the existence of type $j$ obstacle. In equation (2), $j \varepsilon\{1,2,3\}$ characterizes the type of the obstacle and $\phi j=\left\{\left(x_{r}^{(j)}, y_{r}^{(j)}\right)\right.$, $\left.\Theta^{(j)}\right\}$ signifies the obstacle's characteristic and comprises the obstacle shape parameters $\Theta^{(j)}$ (dimensions information) and the obstacle central position $\left(x_{c}^{(j)}, y_{c}^{(j)}\right), e . g$. Wall obstacle has a central position $(\mathrm{Lx} / 2, \mathrm{Ly} / 2),[34]$.

We demonstrated the SS measurements in the field, expending three different types of RF obstacles. Therefore, it can classify and identify the RF characteristics of a particular type of RF obstacle by examining the changes in the SS measurements obtained at diverse locations from different trajectories $[27,28]$. Computer Simulation Technology (CST) is used for the simulation experiment. It is a professional 3D electromagnetic simulation tool [29]. The simulation uses a $60 \mathrm{~mm} \times 60 \mathrm{~mm}$ patch antenna. It sends and receives communication signals and creates interference from purely conductive materials [30].

\section{A. Wall Obstacle}

One of the known obstacles of various dimensions ( 7 x 30 x $30 \mathrm{~cm}^{3}, 10 \times 30 \times 30 \mathrm{~cm}^{3}$, and $15 \times 130 \times 30 \mathrm{~cm}^{3}$ ) are used in the experiments. The RF-SS result in the field is shown in Fig. 4(a) for the $10 \times 30 \times 30 \mathrm{~cm}^{3}$ wall. When the transmitter approaches the wall's edge on one side and the receiver is one meter far on the other side, SS drops down and becomes very low, and vice versa. The SS improves as the receiving or transmitting robot moves away from the obstacle edges, as depicted in Fig. 4(a). The top view of the results is illustrated in Fig. 4(b), where the dark red dots illustrate spikes of Fig. 4(a). Fig. 4(c) depicts various waveforms resulting from the wall obstacles at the receiving robot location for different distances. The waveform reflects the influence of RF obstacles on the RF-SS between robots when the robot moves about the RF obstacle.

\section{B. Cage Obstacle}

A Faraday-like cage is shown in Fig. 5(a). It is made of PEC material. The size of the cage is $30 \times 30 \times 30 \mathrm{~cm}^{3}$. The SS drops and signal expire when a robot is trapped in the cage, as presented in Fig. 5(a). Due to conducting material effects, the SS exhibits oscillating patterns as either antenna approaches the cage opening. The signal drops down when there is no LOS between antennas and becomes weak, as presented in Fig. 5(a). Fig 5(b) is the SS intensity image of Fig. 5(a) and illustrates that the SS goes up as a LOS exists. The effect on RF-SS by the cage obstacle is depicted in Fig. 5(a), 5(b), and 5(c). Different waveforms represent measurement sequences of different trajectories around the obstacle are depicted in Fig. 5(c).

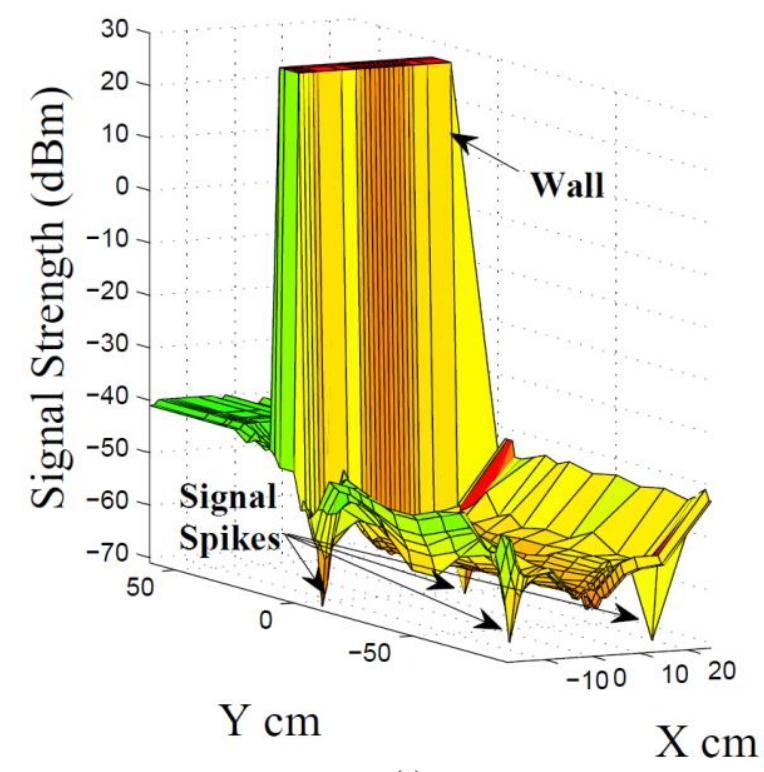

(a)

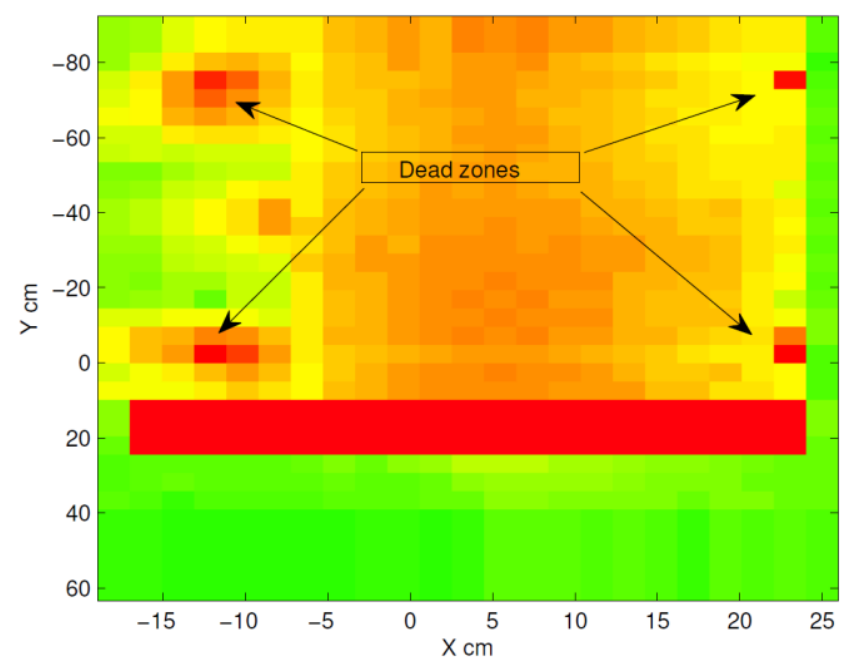

(b)
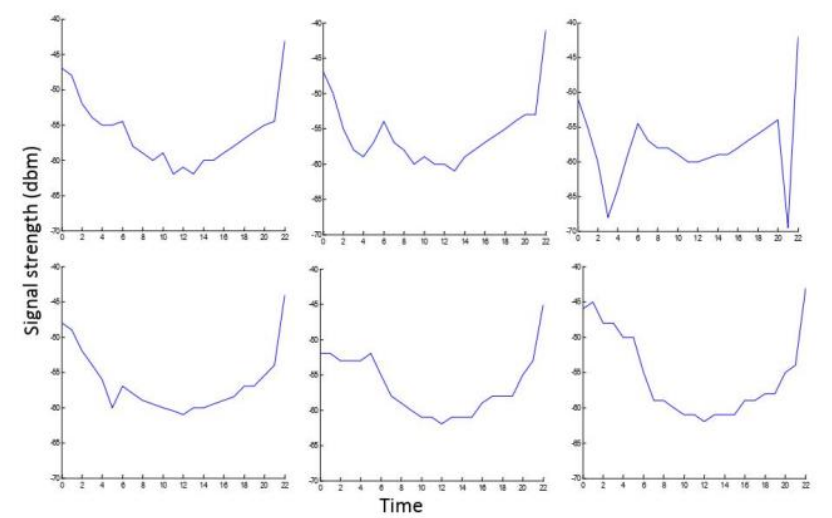

(c)

Fig. 4. Wall Obstacle (a) RF-SS Measurements (b) Top view of (a), and (c) Waveforms for Multiple Trajectories. 


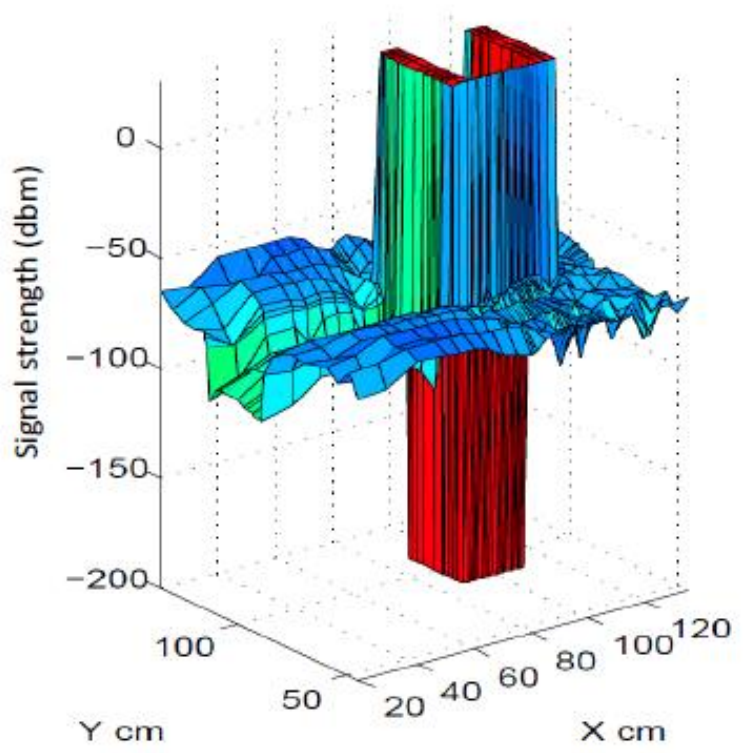

(a)

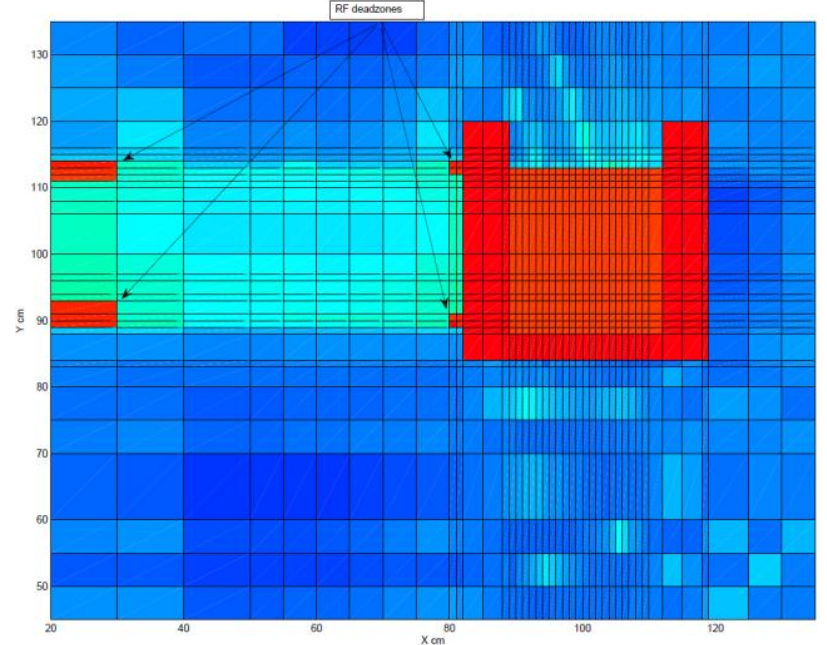

(b)
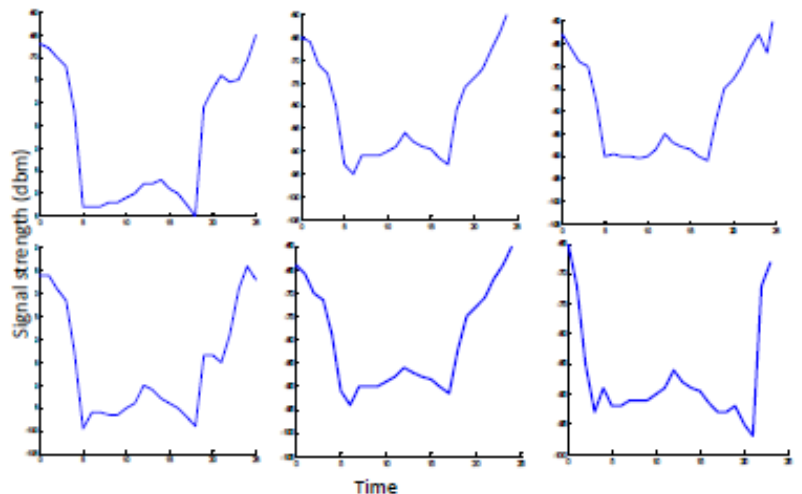

(c)

Fig. 5. Cage Obstacle (a) RF-SS Measurements (b) Top view of (a), and (c) Waveforms for Multiple Trajectories.

\section{Cylinder Obstacle}

Cylinders of radiuses $10 \mathrm{~cm}, 15 \mathrm{~cm}$, and $20 \mathrm{~cm}$ and height of $30 \mathrm{~cm}$ were used in this experiment.
SS measurements simulation results in the field in the existing on an obstacle of $15 \mathrm{~cm}$ diameter centered in the testing area are depicted in Fig. 6(a). The SS dropped down and became unreliable as the receiving robot approached the cylindrical obstacle. Fig. 6(b) presents the SS intensity image of Fig. 6(a).

The influence of the cylinder on the RF-SS is depicted in Fig. 6(a), 6(b), and 6(c). Since the antenna moves in line on either obstacle side, the SS sequence contains enough evidence to identify the obstacle type. When the receiver antenna is close to an HF obstacle, the shadow of the HF obstacle in the HFSS measurement will produce different waveforms for different distances.

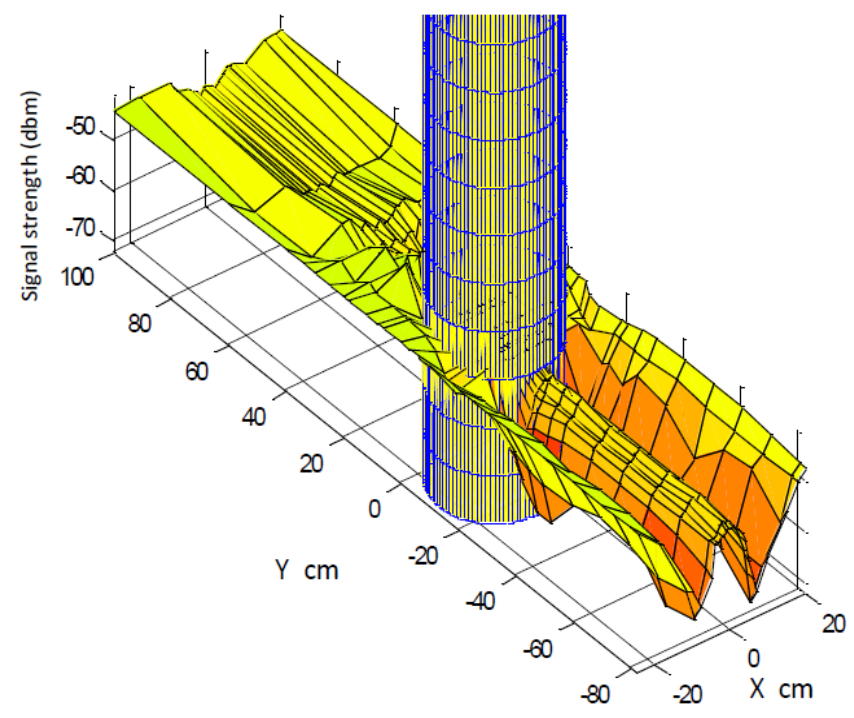

(a)

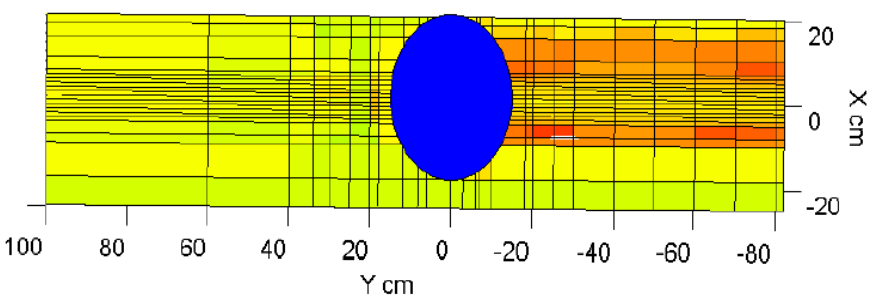

(b)

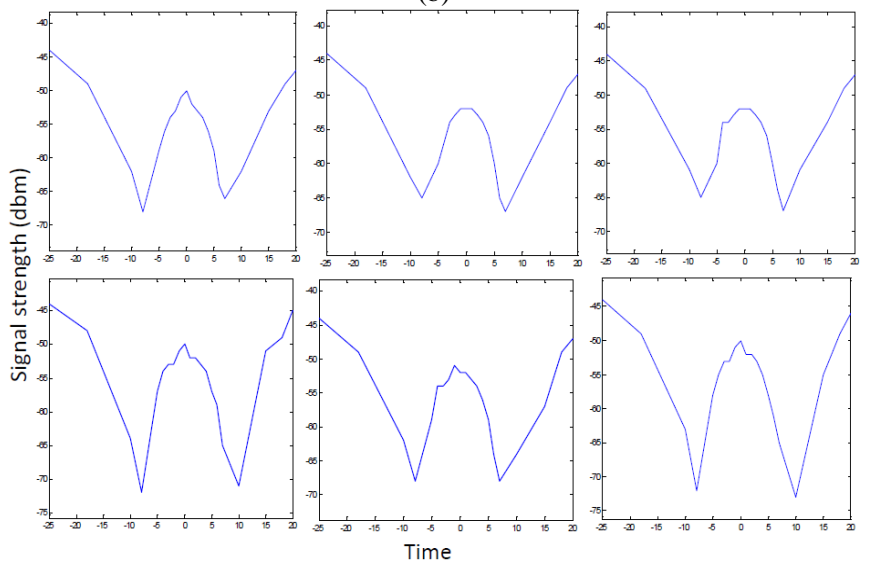

(c)

Fig. 6. Cylinder Obstacle (a) RF-SS Measurements, (b) Top view of Fig. 6, and (c) Waveforms for Multiple Trajectories. 


\section{PhysiCAl EXPERIMENTS FOR MUlTIPLE OBSTACLES}

Conductive known RF obstacles such as cages, walls, and cylinders are created to run multiple physical experiments in the field [27]. Then, we sought a minimum interference environment to run the experiments, and a CC2510 development kit was used. The copper obstacle is centered on the cardboard box in the laboratory space. Then, $2.4 \mathrm{GHz}$ transceivers are moved manually in all directions around the obstacle. We recorded SS measurements at different antenna positions around the obstacle. RF-SS measurements are made on both sides up to $100 \mathrm{~cm}$ in all directions. Next, we made various RF obstacle shapes similar to those used for simulation. The physical results are based on surroundings and floor type. Different materials such as carpets and wood have other effects on the RF signal. Running multiple extensive experiments to choose the best environment leads us to select a box of $15 \mathrm{~cm}$ height for best results [27].

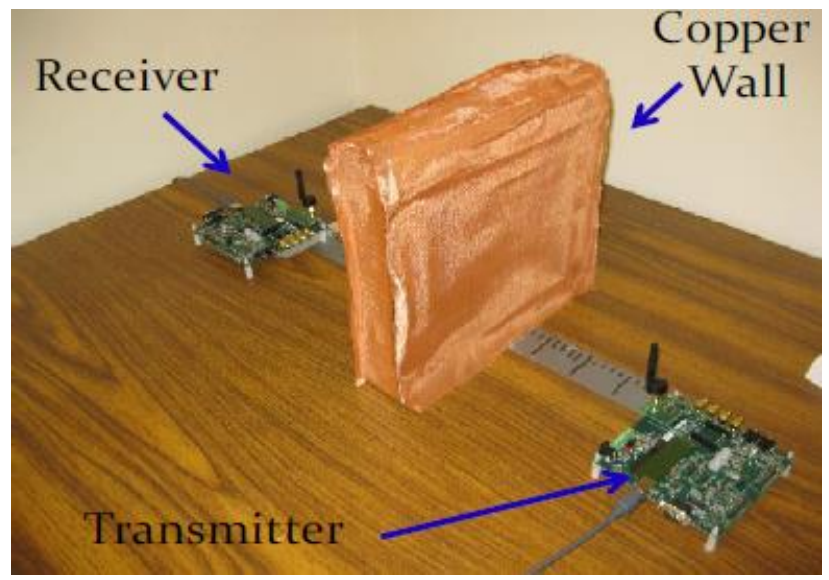

Fig. 7. Copper Wall Obstacle, Receiver and Transmitter.

\section{A. Copper Wall Obstacle}

A wooden box of dimensions $\left(10 \times 30 \times 30 \mathrm{~cm}^{3}\right)$ is created and then covered with a copper screen, as seen in Fig. 7. We collected SS measurements around the obstacle by moving the antennas in different directions. The results are shown in Fig. 8(a). The SS measurements range from high to low, depending on the obstacle effects. The SS turned out to be deficient, as indicated by the spikes in Fig. 8(a). The SS improved when the transceiver was $1 \mathrm{~m}$ apart on one side while the other was still and close to the obstacle, but it remained low as the obstruction prevented LOS.

The physical results presented in Fig. (8) approximates the simulation outcomes shown in Fig. 4(a). The low SS is apparent in Fig. 8(b) that the top view of Fig. 8(a). Therefore, we take advantage of the position of the spikes in estimating the obstacle dimensions, which is valid for simulation results too.

When the transceivers diverge from the obstacle shadow, $\mathrm{SS}$ improves, and it reaches the maximum as the transceivers maintain a LOS. The copper obstacle effects on the SS are depicted in Fig. 8(a)-(c). Therefore, after examining the results of the experiment robots' movements all over the obstacle for multiple straight trajectories, it is clear that the SS contains helpful information that is used to recognize and classify obstacle types. Fig. 8(c) illustrates the signature of the obstacle on the RF signals, and it shows different signal shapes for various trajectories.

\section{B. Copper Cage Obstacle}

A four-sided wood cage of length $=30 \mathrm{~cm}$, width $=30 \mathrm{~cm}$, and height $=30$ is created and covered by a screen of pure copper. The obstacle is centered on a cardboard box that insulates the transceivers from the ground. Next, the transceivers, which preserve a distance of $1 \mathrm{~m}$ apart, are moved around the obstacle. Finally, the transceivers move in all directions outside and inside the cage for SS measurements. Inside the cage, the SS is extremely low and not conducive, as shown in Fig. 9(a).

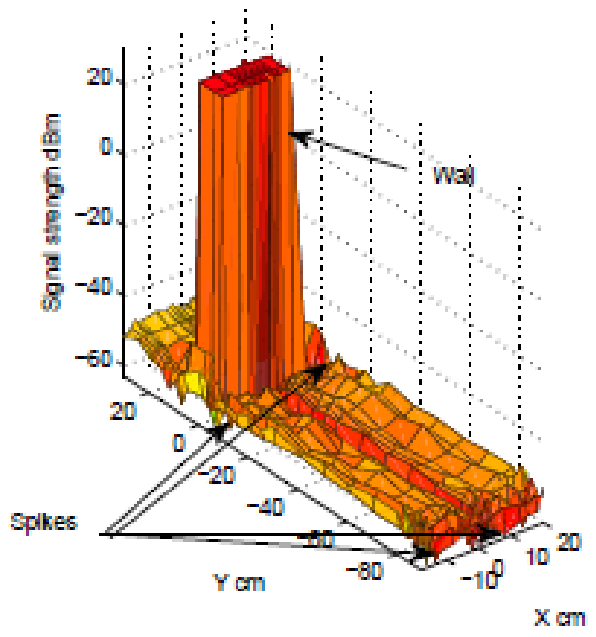

(a)

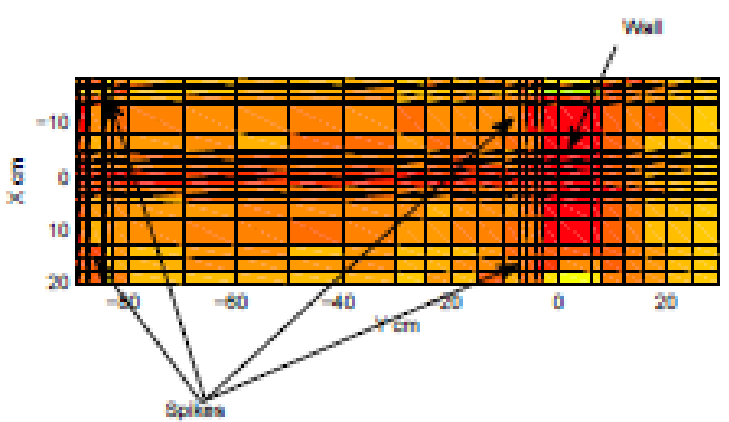

(b)
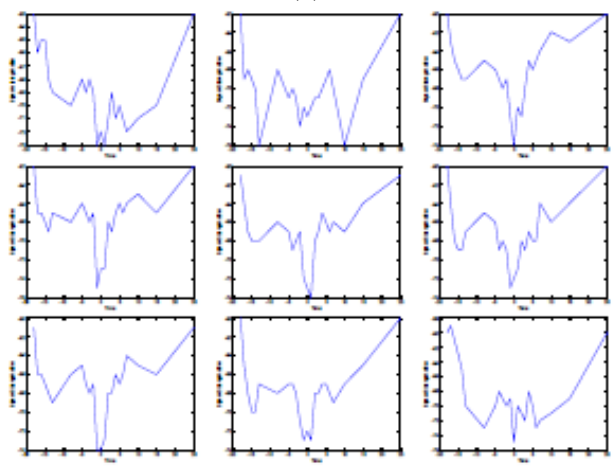

(c)

Fig. 8. Copper Wall Obstacle (a) RF-SS Measurements (b) Top view of Fig. 7(a), and (c) Multiple Signal Shapes. 


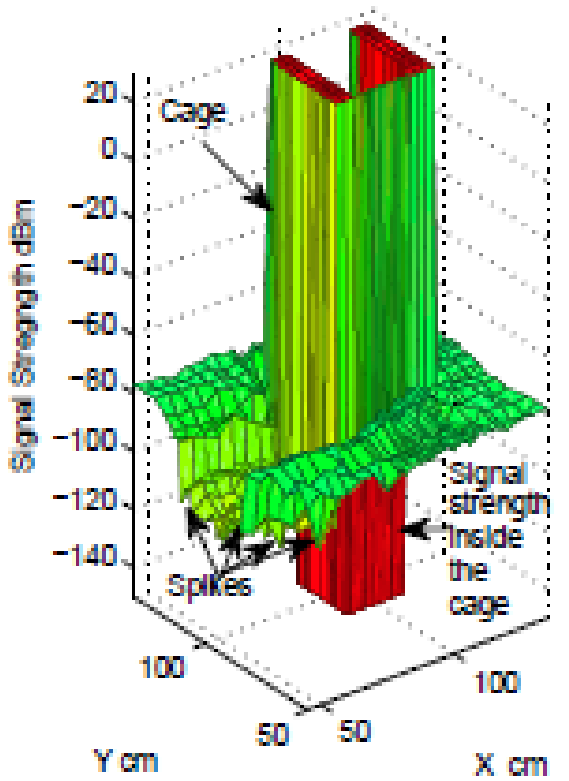

(a)

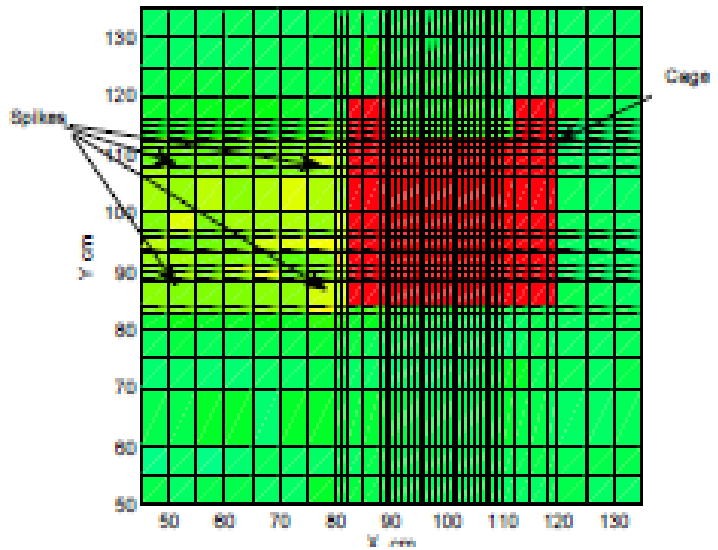

(b)
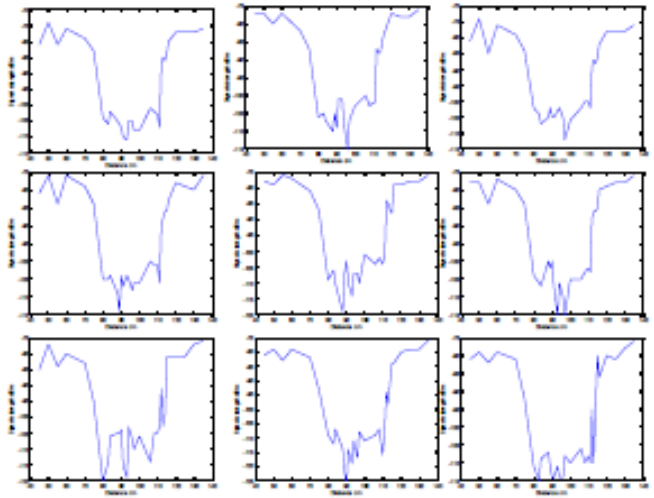

(c)

Fig. 9. Copper Cage Obstacle (a) RF-SS Measurements (b) Top view of Fig. 8(a), and (c) Multiple Signal Shapes.

As a result of LOS loss between the transceivers, the SS dropped down and produced poor conductive connectivity. It is shown in Fig. 9(b), the top view of Fig. 9(a). The SS improves as the transceivers retain the LOS and become more conductive, approximating the simulations as depicted in Fig. 4(a). In Fig. 9(b), the backside of the cage, the SS reaches the maximum conductivity as the transceiver moves further. The results depicted in Fig. 9(a) override the results illustrated in Fig. 5(a) by a value of $-5 \mathrm{~dB}$, resulting from different surrounding electromagnetic sources. Fig. 9(c) illustrates the signature and the obstacle impact on RF-SS, showing different signal shapes for varied trajectories.

\section{VAlidation of PHYSiCAL AND Simulation Results}

The simulation and physical results comparison and validation of the RF obstacle discussed in previous sections are presented. For accuracy and comparison, different signal shapes of the obstacles are plotted in the same graph. Additionally, the effect of various electromagnetic sources on the physical signal shapes is detectable in the signal shapes.

\section{A. Validation of Wall Results}

Using the setup of Fig. 7, multiple wall physical experiments are conducted to demonstrate the simulation results. The experiments are conducted in an environment with fewer interference sources. The transceivers are moved in a bounded area of $2 \mathrm{~m} 2$ around a centered wall obstacle in all directions. The resulting signal shapes for the simulation (black) and physical (red and blue) are depicted in Fig. 10. The signal power difference between the black and red signals ranges from -2 to $-8 \mathrm{dBm}$, while it was above $-15 \mathrm{dBm}$ between black and blue signals due to interference source existence.

\section{B. Validation of Cage Results}

Numerous cage physical experiments are conducted to demonstrate the simulation results. First, the experiments are conducted in an environment with fewer interference sources. Then, the transceivers are moved in a bounded area of $2 \mathrm{~m} 2$ around a centered cage obstacle in all directions.
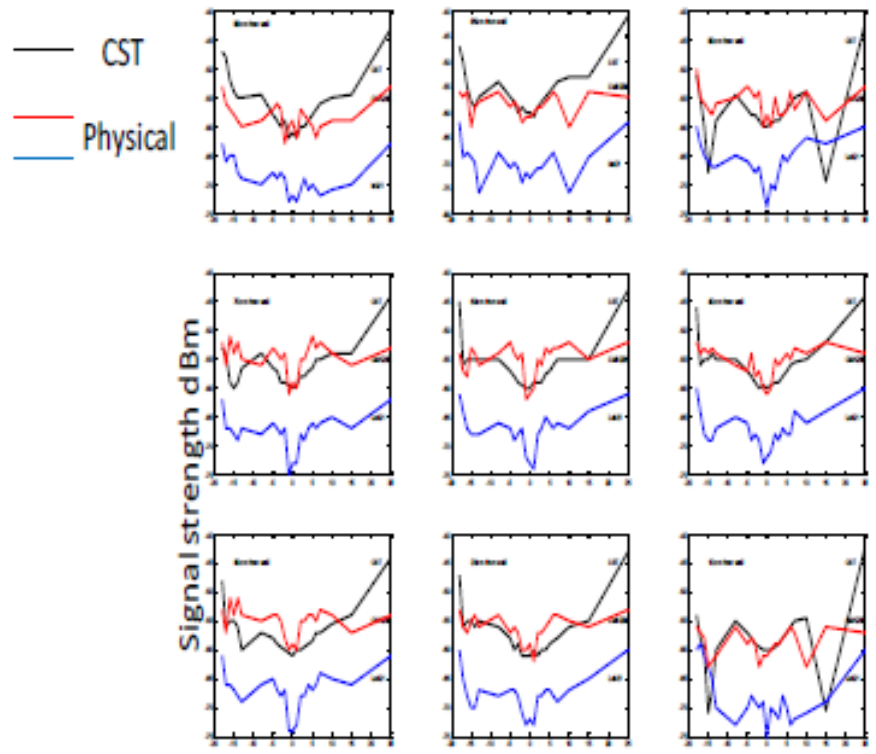

Time

Fig. 10. Wall Simulation and Physical Results Comparison. 
The resulting signal shapes for the simulation (black) and physical (red and blue) are depicted in Fig. 11. The signal power difference between the black and red signals ranges from -3 to $-20 \mathrm{dBm}$, while it was above $-20 \mathrm{dBm}$ between black and blue signals due to interference source existence.

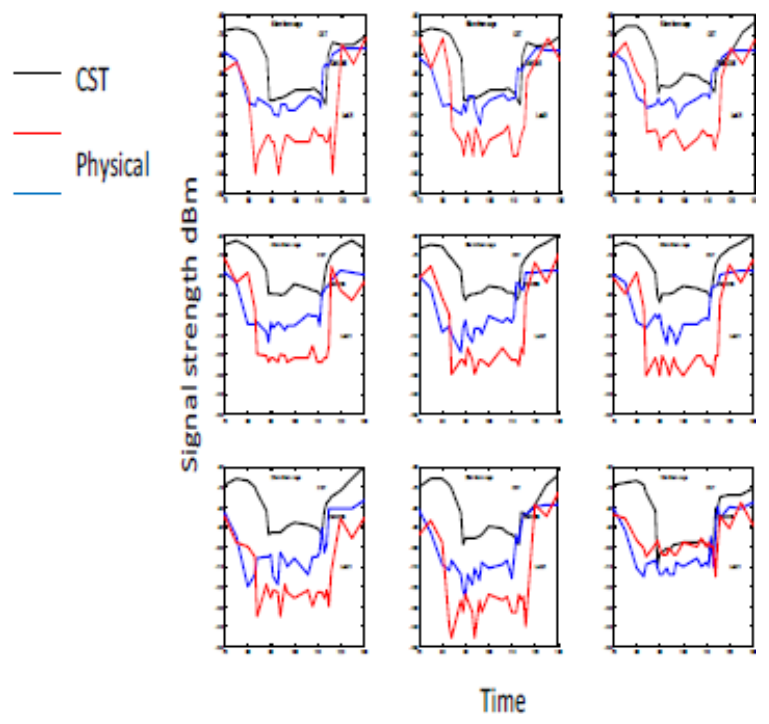

Fig. 11. Cage Obstacle Physical Results Comparison.

\section{OBSTACLE PARAMETERIZATION}

Radio SS propagation is a complicated process. In Sections I and II, we explained that SS is a function of different parameters. In addition, the power of the received SS is a function of how far the transmitter is, obstacles effects, and multipath occurrences such as reflections and refractions $[2,15]$.

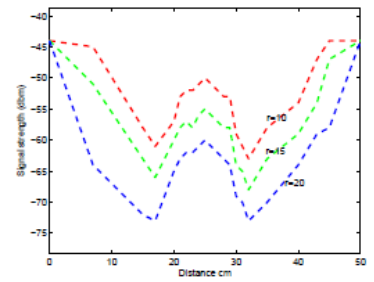

(a)

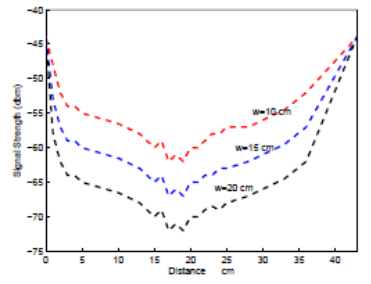

(b)
Fig. 12. Signal shapes for different (a) Different Radiuses (b) Wall widths.

\section{A. Wall Parameterization}

Different RF walls obstacles are used in our experiments with varying sizes of width $\mathrm{w}$. As a result, the wall signal shapes are almost preserved in the "U" shape. The signal shapes are scaled and stretched as the width of the wall increases. Table I shows the average signal strength in $\mathrm{dBm}$ on the robot's trajectories for different wall widths. As the wall width increases by $1 \mathrm{~cm}$, the signal strength average alongside the robot path decreases by $-1.2 \mathrm{dBm}$, as shown in Fig. 12(b).

TABLE I. AVERAGE SS ON THE ROBOT TRAJECTORY

\begin{tabular}{|l|l|l|l|}
\hline Cylinder radius $\mathbf{r}$ & $\mathbf{1 0} \mathbf{c m}$ & $\mathbf{1 5} \mathbf{c m}$ & $\mathbf{2 0} \mathbf{~ c m}$ \\
\hline Average $\mathbf{S S}(\mathbf{d B m})$ & -53 & -59 & -64 \\
\hline
\end{tabular}

\section{RFMR METHOD BASED ON HMM}

The RFMR method is summarized in the significant steps shown in the diagram of Fig. 13. Foremost, in Fig. 14, the measurement vector acquired through multiple robot motion paths is split into various segments (small components) of comparable lengths. Afterward, features are extracted in the frequency domain by applying Fast Fourier Transform (FFT) on every element of the segmented signal. The features components extracted are written to vectors, and then we used a subset of the vectors of features for the training purpose of the created model and the remaining vectors used for model testing. Next, the training subset is clustered to generate observation sequences using the K-mean clustering algorithm [31]. Then, three HMMs models are trained using the generated observation sequences. Each HMM model that contains five states is assigned to each obstacle type. The five states correspond to 5 small segments produced on the robotspecific trajectory. As illustrated, we trained each HMM model using a specific set of observation sequences. Finally, the training set of features is used to train classification models. Accordingly, we accomplished the RFMR method results [28]. Consequently, the results were used by the robot PMCA algorithm that uses the trained HMM results. As a result, we accomplished proactive connectivity [30].

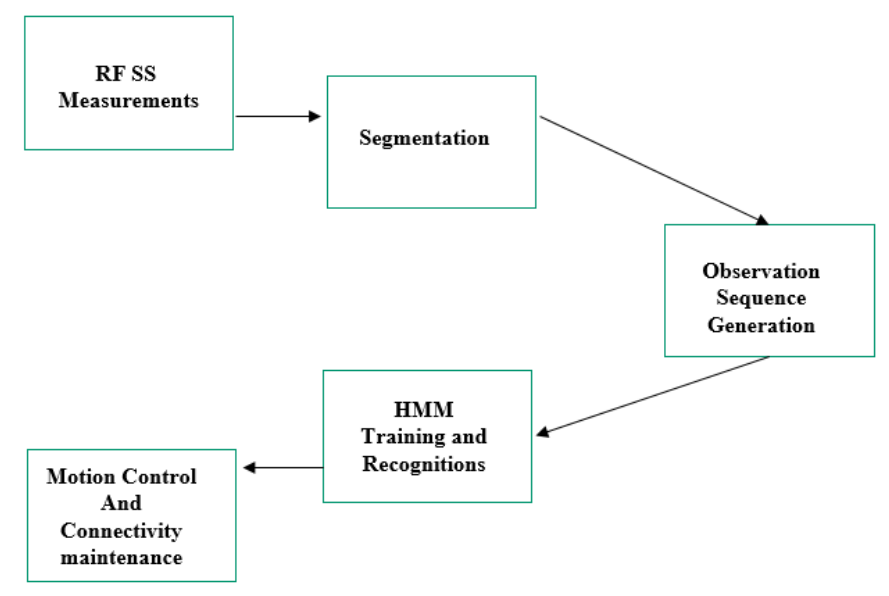

Fig. 13. RFMR Method Block Diagram.

The robot's movement is a sequential event, and our goal is to classify the robot movement in sequential order of the segments. Furthermore, there is a strong analogy between the RFMR method based on HMM results and the word recognition via speech patterns [32]. Therefore, using HMM offers a more spontaneous methodology for RF shadows classification. Naturally, it breakdowns the measurements on the robot's pathway to approximate components comparable to how they were created. However, the HMM method offers a simple technique for classifying segments subset on the robot's path as it moves through an obstacle shadow rather than categorizing the pieces after the obstacle shadow. The HMMs and their application in RFMR are discussed in the following sections. Hence, HMM is a method for stochastic events of a model. Clearly, A model $\lambda$ consists of several states Q, observations B corresponding probabilities, and transitions between states probabilities [11]. Therefore, specified a sequence of observations, $\mathrm{O}$, and $\lambda$ as a model, one 
can obtain $\mathrm{P}(\mathrm{O} \mid \lambda)$. Fundamentally, it is the model representation of the event, and it could be a good or bad representation. To classify data using HMM, we need to create a model $\lambda(\mathrm{j}), \mathrm{j}=1, \ldots, \mathrm{m}_{\mathrm{o}}$, for each class, where $\mathrm{m}_{\mathrm{o}}$ denotes obstacle types number. Then, we must calculate $\mathrm{P}(\mathrm{O} \mid \lambda(\mathrm{j}))$ corresponding to each obstacle type available. Finally, the model with the highest probability is allocated to a novel observation $\mathrm{O}$. Therefore, the obstacle type membership is given to $\mathrm{O}$.

\section{A. Feature Extraction based on SS Measurement Segmentation}

The collected SS measurement vector through the robot moves on the trajectory, $l t h$, is $\beta_{l}^{(j)}=\left[S_{l}^{(j)}(1), S_{l}^{(j)}(2), \ldots\right.$ $\left.S_{l}^{(j)}(N m)\right]^{\mathrm{T}}$, For the $j$ th obstacle type, $N_{m}$ is the SS measurements number on the $l$ th path. Then, as in Fig. 14, each $\beta_{l}^{(j)}$ is segmented into five segments represented as $\alpha_{l, u}^{(j)}=\left[S_{l}^{(j)}((u-1)+1) \ldots S_{l}^{(j)}(5 u)\right]^{T}$, where $u=$ $1,2, \ldots, 5$. Subsequently, a measurement segment $\alpha_{l . u}^{(j)}$ is transformed to the using FFT. Results are represented as $\Gamma_{l, u}^{(j)}=F F T\left(\alpha_{l, u}^{(j)}, N_{F F T}\right), N_{F F T}$ shows the points number in the FFT results. The first ten elements in the FFT result $\Gamma_{l, u}^{(j)}$ are denoted as the feature vector $\gamma_{l, u}^{(j)}=$ $\left[\Gamma_{l, u}^{(j)}(1) \Gamma_{l, u}^{(j)}(2) \ldots \Gamma_{l, u}^{(j)}(10)\right]^{T} \quad$ Of the measurement corresponding to the $l$ th trajectory and $j$ th obstacle type. Once each segment is (j) transferred into frequency space, the feature vector $\Gamma_{l, u}^{(j)}$ is clustered using the K-means clustering algorithm [35].

Then, the HMM uses these binned segments to classify the obstacle shadow based on the probabilistic sequence of segments. Our numerical experiments tried different training sets to examine their effect on the recognition rate. We found that the recognition rate is affected positively by the size increase of the training sets. Data were randomly split into training and testing sets to verify the HMM classifier [35]. We randomly select $60 \%$ of the measurement vectors into the training set $S_{\text {train }}^{c}$ which is used for c clustering and training, and the rest constitutes the testing set $S_{\text {test }}^{c}$.

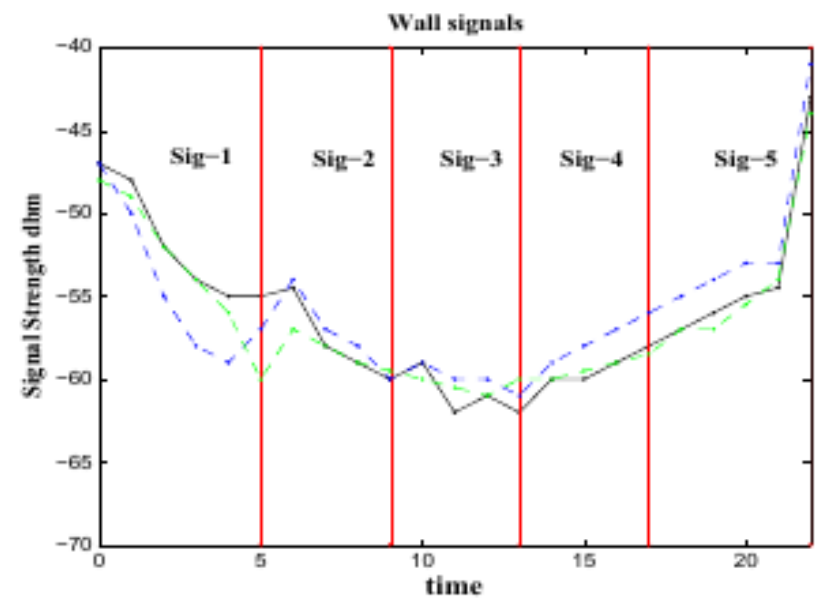

Fig. 14. One Segmented Wall Signal.

\section{B. The Generation of Observation Sequences}

The collected vectors $\gamma_{l, u}^{(j)}$ in the model training set $S_{\text {train }}^{c}$ are split into $G$ different clusters using the k-means algorithm. The $G$ clusters are arranged as $D_{1}, D_{2}, \cdots, D_{G}$. Therefore, we can minimize the within-cluster sum of squares (WCSS). Equation (3) presents the $k$-means algorithm stages, and it is written as.

$\arg \min _{D 1, \cdots, D G} \quad \sum_{g=1}^{G} \sum_{\beta_{l}^{(j)} \in S_{\text {train }}^{c}, \gamma_{l, u}^{(j)} \in D g}\left\|\gamma_{l, u}^{(j)}-\mu_{\mathrm{g}}\right\|^{2}$

where the parameter $\mu_{g}$ represents the centroid of $D_{g}$, i.e., the points mean in $D_{g},\left\|\gamma_{l, u}^{(j)}-\mu_{\mathrm{g}}\right\|^{2}$ represents the vector $\gamma_{l, u}^{(j)}$ and $\mu_{g}$ distance separation. After the $\mathrm{k}$-means clustering algorithm generates $D_{g}$ and $\mu_{g}$, it allocates observation symbols to the feature vectors. As a result, the observation sequences for HMM training and test sets are produced. Initially, The $C=\left\{C_{l}, \cdots, C_{G}\right\}$ with $C_{g}$ as the $g$ th symbol signifies the symbol set of HMM observations [34]. The $C_{l, u}^{(j)}$ symbol conforming to the data segment $\gamma_{l, u}^{(j)}$ is allocated to the value $C_{g}$ if $\left\|\gamma_{l, u}^{(j)}-\mu_{\mathrm{g}}\right\|^{2}$ has the minimum value in the set, $\mathrm{g}$ $\in\{1,2, \cdots, \mathrm{G}\}$. Explicitly, $C_{l, u}^{(j)}$ is allocated to symbol $C_{g}$ when $\mu_{g}$ is the closer centroid to the feature vector $\gamma_{l, u}^{(j)}$. The $C_{l, u}^{(j)}$ vectors of the $l$ th trajectory segments are concatenated to form the vector $C_{l}^{(j)}=\left[\begin{array}{llll}C_{l, 1}^{(j)} & \ldots & C_{l, 5}^{(j)}\end{array}\right]^{T}$ [34]. The resulting vector $C_{l}^{(j)}$ is the observation sequence corresponding to the measurement vector $\beta_{l}^{(j)}$. The HMM training set $S_{\text {train }}^{\text {HMM }}$ conforming vector $\beta_{l}^{(j)}$ contains the observation sequence $C_{l}^{(j)}$ is included in the training set $S_{\text {train }}^{c}$; otherwise, it is in the test set $S_{\text {test }}^{H M M}$.

In conclusion, applying the mentioned procedure, for the trajectory, lth, in the existence of type $j$ obstacle, the vector $\beta_{l}^{(j)}$ is segmented into segments $\alpha_{l, u}^{(j)}, u=1,2,3,4,5$. Consequentially, each $\alpha_{l, u}^{(j)}$ is converted by FFT, and the FFT result is symbolized by $\Gamma_{l, u}^{(j)}$. The first ten elements in $\Gamma_{l, u}^{(j)}$ are selected to form the feature vector $\gamma_{l, u}^{(j)}$. The feature vectors are clustered using the $k$-means algorithm to generate $G$ clusters, $D_{l}, \cdots, D_{G}$, and the corresponding cluster centroids $\mu_{1}, \ldots, \mu_{G}$.

The individual segment feature vector $\gamma_{l, u}^{(j)}$ is consigned with a symbol $C_{l, u}^{(j)} \in C$ by the parameters of the cluster [35]. Then, the observation sequence $C_{l}^{(j)}$ is created by concatenating the $C_{l, u}^{(j)}$ vector [34]. Finally, the observation sequence $C_{l}^{(j)}$ is created from each trajectory $\beta_{l}^{(j)}$ vector and ready for training or testing HMMs models [34, 35].

\section{RFMR BASED HMM NUMERICAL RESULTS}

The HMM training set $S_{\text {test }}^{H M M}$ resulted from the previous section will be used for training HMMS models. Three HMMs, models, $\lambda(j)$, with $j=1,2,3$, conforming wall, cage, and cylinder obstacles, are trained for classification. 
Assumed an observation sequence $\mathrm{C}_{1}^{(\mathrm{j})}$, is comprised of numerous observation symbols. Next, given the HMM $\lambda(p)$, the $\mathrm{P}(\mathrm{Cl} \mid \lambda(\mathrm{p}))$ is a conditional probability of $\mathrm{C}_{1}^{(\mathrm{j})}$. For the classification process, the $\mathrm{P}(\mathrm{Cl} \mid \lambda(\mathrm{p}))$, can be calculated for the parameter $p=1,2,3$. When the value $p=\hat{p}$, the maximum probability $\mathrm{P}\left(\mathrm{C}_{\mid} \mid \lambda_{(\mathrm{p})}\right)$ is achieved, and the obstacle of type $\hat{\mathrm{p}}$ is the prediction of RFMR method results.

Moreover, if a transceiver approaches an obstacle while the complete observation sequence was not existing, the first few observations for the classification.is found in the observation sequence of a variable-length vector $\mathrm{C}_{1}^{(\mathrm{j})}$.

\section{A. Examining Cylinder Obstacle of different Radius}

The total measurement vectors used are 535. A set of 321 vectors is for the training, and the rest is for testing. These measurement vectors contain data from three different cylinder radiuses, $10 \mathrm{~cm}, 15 \mathrm{~cm}$, and $20 \mathrm{~cm}$, with a height of $30 \mathrm{~cm}$. The confusion matrix $(\mathrm{CM})$ that signifies the RFMR results is depicted in Tables II, III, and IV, each row of the $\mathrm{CM}$ denotes the predicted class. Table II establishes the CM using the first two segments of observation sequences; a percentage of $88 \%$ was the classification rate attained. Table III reflects the CM using the first three segments, and the rate was $95 \%$. Finally, a rate of $100 \%$ was reached using four segments as presented in Table IV. The results are improved for the HMM classifier as the number of segments increases, and consequently, the rates become reliable.

\section{B. Examining Wall Obstacle of Various Dimensions}

The total measurement vectors used are 455 . A subset of two hundred seventy-three vectors is for the training, and the rest is for testing. The measurement vectors contain data for 7 $\times 30 \times 30 \mathrm{~cm}^{3}, 10 \times 30 \times 30 \mathrm{~cm}^{3}$, and $15 \times 30 \times 30 \mathrm{~cm}^{3}$ wall dimensions. The CM of the RFMR results is shown in Tables V, VI, and VII. Table V demonstrates the CM of RFMR of wall measurement vectors based on the first two segments, and the rate was $70 \%$.

In comparison, Table VI presents the $\mathrm{CM}$ with the first three segments, and the rate was $77 \%$. Finally, Table VII validates the CM of RFMR results based on the first four segments; the success rate was $93 \%$. The results are improved for the HMM classifier as the number of segments increases, and consequently, the rates become excellent.

TABLE II. CM OF RFMR FOR CYLINDER USING 2 OBSERVATIONS

\begin{tabular}{|l|l|l|l|}
\hline Cylinder radius r & $\mathbf{1 0} \mathbf{~ c m}$ & $\mathbf{1 5} \mathbf{c m}$ & $\mathbf{2 0} \mathbf{~ c m}$ \\
\hline $\mathbf{1 0} \mathbf{~ c m}$ & 1 & 0.0 & 0.14 \\
\hline $\mathbf{1 5} \mathbf{~ c m}$ & 0.0 & 1 & 0.22 \\
\hline $\mathbf{2 0} \mathbf{~ c m}$ & 0.0 & 0.0 & 0.64 \\
\hline
\end{tabular}

TABLE III. CM OF RFMR FOR CYLINDER USING 3 OBSERVATIONS

\begin{tabular}{|l|l|l|l|}
\hline Cylinder radius $\mathbf{r}$ & $\mathbf{1 0} \mathbf{~ c m}$ & $\mathbf{1 5} \mathbf{c m}$ & $\mathbf{2 0} \mathbf{~ c m}$ \\
\hline $\mathbf{1 0} \mathbf{~ c m}$ & 0.86 & 0.0 & 0.0 \\
\hline $\mathbf{1 5} \mathbf{~ c m}$ & 0.14 & 1 & 0.0 \\
\hline $\mathbf{2 0} \mathbf{~ c m}$ & 0.0 & 0.0 & 1 \\
\hline
\end{tabular}

TABLE IV. CM OF RFMR FOR CYLINDER USING 4 OBSERVATIONS

\begin{tabular}{|l|l|l|l|}
\hline Cylinder radius $\mathbf{r}$ & $\mathbf{1 0} \mathbf{~ c m}$ & $\mathbf{1 5} \mathbf{c m}$ & $\mathbf{2 0} \mathbf{~ c m}$ \\
\hline $\mathbf{1 0} \mathbf{~ c m}$ & 1 & 0.0 & 0.0 \\
\hline $\mathbf{1 5} \mathbf{~ c m}$ & 0.0 & 1 & 0.0 \\
\hline $\mathbf{2 0} \mathbf{~ c m}$ & 0.0 & 0.0 & 1 \\
\hline
\end{tabular}

TABLE V. CM OF RFMR FOR WALL USING 2 OBSERVATIONS

\begin{tabular}{|l|l|l|l|}
\hline Wall width (w) & $\mathbf{7} \mathbf{~ c m}$ & $\mathbf{1 0} \mathbf{~ c m}$ & $\mathbf{1 5} \mathbf{~ c m}$ \\
\hline $\mathbf{7} \mathbf{~ c m}$ & 0.85 & 0.46 & 0.0 \\
\hline $\mathbf{1 0} \mathbf{~ c m}$ & 0.15 & 0.54 & 0.31 \\
\hline $\mathbf{1 5} \mathbf{~ c m}$ & 0.0 & 0.0 & 0.69 \\
\hline
\end{tabular}

TABLE VI. CM OF RFMR FOR WALL USING 3 OBSERVATIONS

\begin{tabular}{|l|l|l|l|}
\hline Wall width (w) & $\mathbf{7} \mathbf{~ c m ~}$ & $\mathbf{1 0} \mathbf{~ c m}$ & $\mathbf{1 5} \mathbf{~ c m}$ \\
\hline $\mathbf{7} \mathbf{~ c m}$ & 0.87 & 0.44 & 0.0 \\
\hline $\mathbf{1 0} \mathbf{~ c m}$ & 0.13 & 0.56 & 0.16 \\
\hline $\mathbf{1 5} \mathbf{~ c m}$ & 0.0 & 0.0 & 0.84 \\
\hline
\end{tabular}

TABLE VII. CM OF RFMR FOR WALL USING 4 OBSERVATIONS

\begin{tabular}{|l|l|l|l|}
\hline Wall width (w) & $\mathbf{7} \mathbf{~ c m}$ & $\mathbf{1 0} \mathbf{~ c m}$ & $\mathbf{1 5} \mathbf{~ c m}$ \\
\hline $\mathbf{7} \mathbf{~ c m}$ & 0.95 & 0.08 & 0.0 \\
\hline $\mathbf{1 0} \mathbf{~ c m}$ & 0.05 & 0.92 & 0.12 \\
\hline $\mathbf{1 5} \mathbf{~ c m}$ & 0.0 & 0.0 & 0.88 \\
\hline
\end{tabular}

TABLE VIII. CM OF RFMR FOR ALL OBSTACLES USING 2 OBSERVATIONS

\begin{tabular}{|l|l|l|l|l|l|}
\hline $\begin{array}{l}\text { Different } \\
\text { Obstacle }\end{array}$ & $\begin{array}{l}\text { Cage } \\
\mathbf{3 0} \mathbf{~ c m}^{3}\end{array}$ & $\begin{array}{l}\text { Wall } \\
\mathbf{1 0} \mathbf{~ c m}\end{array}$ & $\begin{array}{l}\text { Wall } \\
\mathbf{1 5} \mathbf{~ c m}\end{array}$ & $\begin{array}{l}\text { Cylinder } \\
\mathbf{1 0} \mathbf{~ c m}\end{array}$ & $\begin{array}{l}\text { Cylinder } \\
\mathbf{1 5} \mathbf{~ c m}\end{array}$ \\
\hline $\begin{array}{l}\text { Cage } \\
\mathbf{3 0} \mathbf{~ c m}^{3}\end{array}$ & 1 & 0.0 & 0.0 & 0.0 & 0.0 \\
\hline $\begin{array}{l}\text { Wall } \\
\mathbf{w}=\mathbf{1 0} \mathbf{~ c m}\end{array}$ & 0.0 & 1 & 0.44 & 0.00 & 0.0 \\
\hline $\begin{array}{l}\text { Wall } \\
\mathbf{w}=\mathbf{1 5} \mathbf{~ c m}\end{array}$ & 0.0 & 0.0 & 0.56 & 0.0 & 0.0 \\
\hline $\begin{array}{l}\text { Cylinder } \\
\mathbf{r = 1 0} \mathbf{~ c m}\end{array}$ & 0.0 & 0.0 & 0.00 & 0.80 & 0.0 \\
\hline $\begin{array}{l}\text { Cylinder } \\
\mathbf{r = 1 5} \mathbf{~ c m}\end{array}$ & 0.0 & 0.0 & 0.0 & 0.20 & 1 \\
\hline
\end{tabular}

\section{Examining Walls, Cages and Cylinders Obstacle of} Different Sizes

In the experiment that combines three different size obstacles, the total measurement vectors used are 825 . Four hundred ninety-five vectors are the training set and the rest for testing. Tables VIII, IX, and X illustrate the CM of the RFMR results for all combined obstacles observation vectors where the predicted class is expressed by $\mathrm{CM}$ rows approximated to the actual class. Table VIII establishes the CM of RFMR results using the first two segments, and the classification rate is $87 \%$. When increasing the segment number to three, the success rate was $89 \%$, as shown in Table IX. Ultimately, the classification rate increases and reaches $92 \%$ as the segments number increased to four and above, as illustrated in Table X. 
TABLE IX. CM OF RFMR ALL OBSTACLES USING 3 OBSERVATIONS

\begin{tabular}{|l|l|l|l|l|l|}
\hline $\begin{array}{l}\text { Cage } \\
\mathbf{3 0} \mathbf{~ c m}^{\mathbf{3}}\end{array}$ & 1 & 0.0 & 0.0 & 0.0 & 0.0 \\
\hline $\begin{array}{l}\text { Wall } \\
\mathbf{w}=\mathbf{1 0} \mathbf{~ c m}\end{array}$ & 0.0 & 0.34 & 0.0 & 0.00 & 0.0 \\
\hline $\begin{array}{l}\text { Wall } \\
\mathbf{w}=\mathbf{1 5} \mathbf{~ c m}\end{array}$ & 0.0 & 0.66 & 0.44 & 1 & 0.0 \\
\hline $\begin{array}{l}\text { Cylinder } \\
\mathbf{r = 1 0} \mathbf{~ c m}\end{array}$ & 0.0 & 0.0 & 0.56 & 0.80 & 0.0 \\
\hline $\begin{array}{l}\text { Cylinder } \\
\mathbf{r = 1 5} \mathbf{~ c m}\end{array}$ & 0.0 & 0.0 & 0.0 & 0.20 & 1 \\
\hline
\end{tabular}

TABLE X. CM OF RFMR FOR ALL OBSTACLES USING 4 OBSERVATIONS

\begin{tabular}{|l|l|l|l|l|l|}
\hline $\begin{array}{l}\text { Different } \\
\text { Obstacle }\end{array}$ & $\begin{array}{l}\text { Cage } \\
\mathbf{3 0} \mathbf{~ c m}^{\mathbf{3}}\end{array}$ & $\begin{array}{l}\text { Wall } \\
\mathbf{1 0} \mathbf{~ c m}\end{array}$ & $\begin{array}{l}\text { Wall } \\
\mathbf{1 5} \mathbf{~ c m}\end{array}$ & $\begin{array}{l}\text { Cylinder } \\
\mathbf{1 0} \mathbf{~ c m}\end{array}$ & $\begin{array}{l}\text { Cylinder } \\
\mathbf{1 5} \mathbf{~ c m}\end{array}$ \\
\hline $\begin{array}{l}\text { Cage } \\
\mathbf{3 0} \mathbf{~ c m}^{\mathbf{3}}\end{array}$ & 1 & 0.0 & 0.0 & 0.0 & 0.0 \\
\hline $\begin{array}{l}\text { Wall } \\
\mathbf{w}=\mathbf{1 0} \mathbf{~ c m}\end{array}$ & 0.0 & 1 & 0.40 & 0.00 & 0.0 \\
\hline $\begin{array}{l}\text { Wall } \\
\mathbf{w}=\mathbf{1 5} \mathbf{~ c m}\end{array}$ & 0.0 & 0.0 & 0.60 & 0.0 & 0.0 \\
\hline $\begin{array}{l}\text { Cylinder } \\
\mathbf{r = 1 0} \mathbf{~ c m}\end{array}$ & 0.0 & 0.0 & 0.0 & 1 & 0.0 \\
\hline $\begin{array}{l}\text { Cylinder } \\
\mathbf{r = 1 5} \mathbf{~ c m}\end{array}$ & 0.0 & 0.0 & 0.0 & 0.00 & 1 \\
\hline
\end{tabular}

In conclusion, the results are improved for the HMM classifier as the number of observation segments increases, and consequently, the rates of successful classification become promising and outstanding. Therefore, it proves that the proposed methods are reliable for the best classification rates, thus, achieving proactive robot control in the field.

\section{Proactive Motion Control Algorithm (PMCA) FOR PRESERVING CONNECTIVITY}

The developing application of mobile robotics networks has produced the control motion concept of mobile nodes communication, so nodes can preserve connectivity while finishing their tasks in the field $[8,34]$. However, the control motion techniques require deep exploration and the creation of more reliable algorithms in the robotic field [7]. When the SS drops down in the field, and a robot loses the collaborating robots in the swarm, it starts preserving connectivity through the movement control algorithm, which assists the robot in reaching a location in the field, where it can gain coverage communicate with other team members. Depending on the results of the RFMR method, the proposed PCMA algorithm decides either to continue the current trajectory or backward movement until it retains reliable SS. The PCMA control decision is mainly based on the information learned from the obstacle shadow recognition.

The proactive control motion algorithm has two choices to achieve its motion control of mobile robots. As mentioned earlier, the control choices are based on the information learned from RFMA results. Firstly, the PMCA can continue to move robots in the current trajectory across the obstacle until the robots preserve communication successfully. The second control choice is moving the robot backward and computing a 4-D gradient based on SS to define the strong SS direction and then communicate with the team [33]. In summary, algorithm one and the flowchart of PCMA in Fig. 15 illustrate the actual steps to control the mobile robot motion to preserve communication.
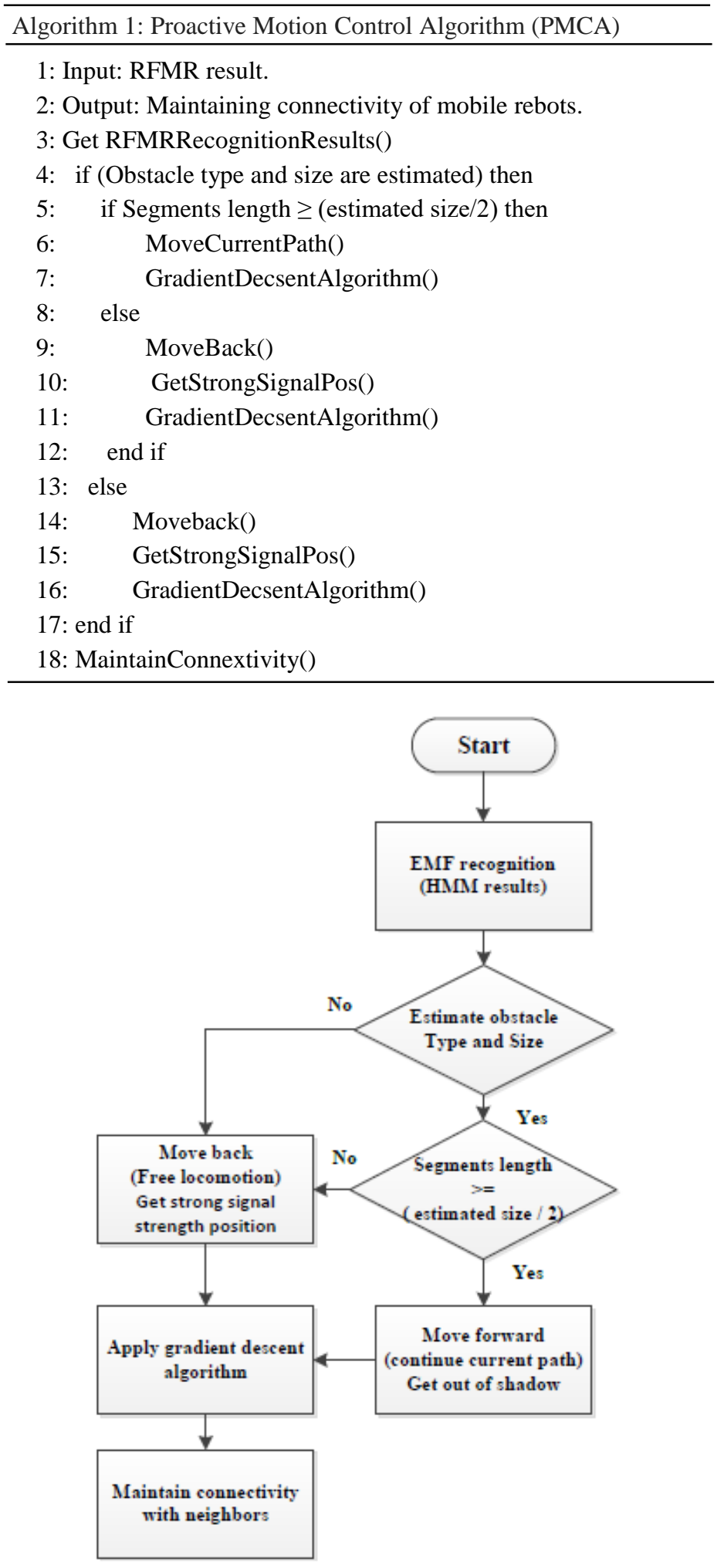

Fig. 15. The PMCA Flow Chart. 


\section{Gradient-BASEd on the Proactive CONTROL ALGORITHM}

The gradient computation process in the field is based on measuring SS between to mobile transceivers that are at $d$ distance apart as in Fig. 20, where the SS is measured at the receiver side. The signal $S_{l}^{(j)}(t)$ at time t of the lth trajectory around an obstacle type $j$ is calculated according to Equation (2). When mobile robots move and retain $\operatorname{LOS}, S_{l}^{(j)}(t)$ is stable and preserves robots connectivity. In contrast, the SS dropped as a conductive obstacle blocks the moving robots [34].

Accordingly, the signal measurements, $S_{l}^{(j)}(t)$, collected through the robot's motion at the position $\left(x_{t}^{(i)}, y_{t}^{(i)}\right), i=1,2$ at time $t$. Next, the gradient is calculated for a specific robot trajectory [34]. For the $l$ th trajectory, the gradient vector can be expressed as

$\nabla S_{l}^{(j)}(t)=\left[\frac{\partial S_{l}^{(j)}(t)}{\partial x_{t}^{(1)}} \frac{\partial S_{l}^{(j)}(t)}{\partial y_{t}^{(1)}} \frac{\partial S_{l}^{(j)}(t)}{\partial x_{t}^{(2)}} \frac{\partial S_{l}^{(j)}(t)}{\partial y_{t}^{(2)}}\right]^{T}$

Robot1

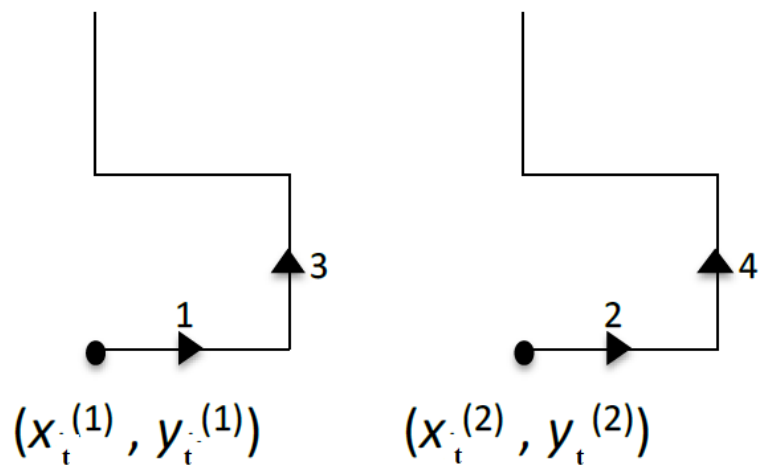

Fig. 16. The Transmitter and Receiver Robot Stepwise Trajectory.

The SS gradient is calculated following the of Fig. 16, the initial position at time $t$ for robot one and robot two are $\left(x_{t}^{(1)}, y_{t}^{(1)}\right)$ and $\left.x_{t}^{(2)}, y_{t}^{(2)}\right)$. As explained in the method below, we assumed that one robot is moving while the other stays still to compute the gradient. As pointed out in Fig. 16, during time $\mathrm{t}$ and $\mathrm{t}+1$, robot one moves along trajectory segment 1 , so $x_{t+1}^{(1)}=x_{t}^{(1)}+\Delta x, y_{t+1}^{(1)}=y_{t}^{(1)}, x_{t+1}^{(2)}=x_{t}^{(2)}, y_{t+1}^{(2)}=y_{t}^{(2)}$, and the gradient element $\frac{\partial S_{l}^{(j)}(t)}{\partial x_{t}^{(1)}}$ is computed a.

$\frac{\partial S_{l}^{(j)}(t)}{\partial x_{t}^{(1)}} \approx \frac{\partial S_{l}^{(j)}(t)}{\partial x_{t}^{(1)}}=\frac{S_{l}^{(j)}(t+1)-S_{l}^{(j)}(t)}{\Delta x}$

Throughout time $\mathrm{t}+1$ and $\mathrm{t}+2$, robot two moves along segment 2 , so $x_{t+2}^{(1)}=x_{t+1}^{(1)}, y_{t+2}^{(1)}=y_{t+1}^{(1)}, x_{t+2}^{(2)}=x_{t+1}^{(2)}+\Delta x$, $y_{t+2}^{(2)}=y_{t+1}^{(2)}$ And the gradient element $\frac{\partial s_{l}^{(j)}(t)}{\partial x_{t}^{(2)}}$ is computed as.

$\frac{\partial S_{l}^{(j)}(t)}{\partial x_{t}^{(2)}} \approx \frac{\partial S_{l}^{(j)}(t+1)}{\partial x_{t+1}^{(2)}}=\frac{s_{l}^{(j)}(t+2)-s_{l}^{(j)}(t+1)}{\Delta x}$
In time $\mathrm{t}+2$ and $\mathrm{t}+3$, robot one moves along segment 3 , so $x_{t+3}^{(1)}=x_{t+2}^{(1)}, y_{t+3}^{(1)}=y_{t+2}^{(1)}+\Delta y, x_{t+3}^{(2)}=x_{t+2}^{(2)}, y_{t+2}^{(2)}=y_{t+1}^{(2)}$, and the gradient element $\frac{\partial s_{l}^{(j)}(t)}{\partial x_{t}^{(2)}}$ is computed as.

$\frac{\partial s_{l}^{(j)}(t)}{\partial y_{t}^{(1)}} \approx \frac{\partial s_{l}^{(j)}(t+2)}{\partial y_{t+2}^{(2)}}=\frac{s_{l}^{(j)}(t+3)-s_{l}^{(j)}(t+2)}{\Delta y}$

In time $\mathrm{t}+3$ and $\mathrm{t}+4$, robot two moves along trajectory segment 4 , so $x_{t+4}^{(1)}=x_{t+3}^{(1)}, y_{t+4}^{(1)}=y_{t+3}^{(1)}, x_{t+3}^{(2)}=x_{t+2}^{(2)}, y_{t+4}^{(2)}=$ $y_{t+3}^{(2)}+\Delta y$, and the gradient element $\frac{\partial s_{l}^{(j)}(t)}{\partial x_{t}^{(2)}}$ is computed as.

$\frac{\partial s_{l}^{(j)}(t)}{\partial y_{t}^{(2)}} \approx \frac{\partial s_{l}^{(j)}(t+3)}{\partial y_{t+3}^{(2)}}=\frac{s_{l}^{(j)}(t+4)-s_{l}^{(j)}(t+3)}{\Delta y}$

As shown in Fig. 17, arrows indicate the gradient direction, and yellow grids illustrate reliable SS due to LOS existence between the communicated transceivers. The gradient strength and direction depend on the robot's location concerning the obstacle position in the field. For example, if one robot is surrounded inside the cage, the gradient drops down as green boxes indicate. Accordingly, any movement for the outer robot did not improve the SS for communication. However, when the robot in the cage changes position, the SS improves enough for communication, as in Fig. 17.

The scenario of Fig. 18 illustrates an obstacle and two robots in the simulation field. One robot moves in a stepwise trajectory, and the other is stays still. Consequently, the gradient is scattered when no LOS exists and does not contain useful information due to obstacle shadow. However, the gradient improved as the LOS became clear.

The scenario of Fig. 19 illustrates an obstacle and two robots in the simulation field. One robot stays still closer to the obstacle corner while the robot moves through a stepwise trajectory. The gradient is scattered when no LOS exists and does not contain useful information due to obstacle shadow. However, the gradient improved as the LOS became clear. In summary, the gradient helps find the right direction of the partner, as illustrated by Fig. 18 and Fig. 19.

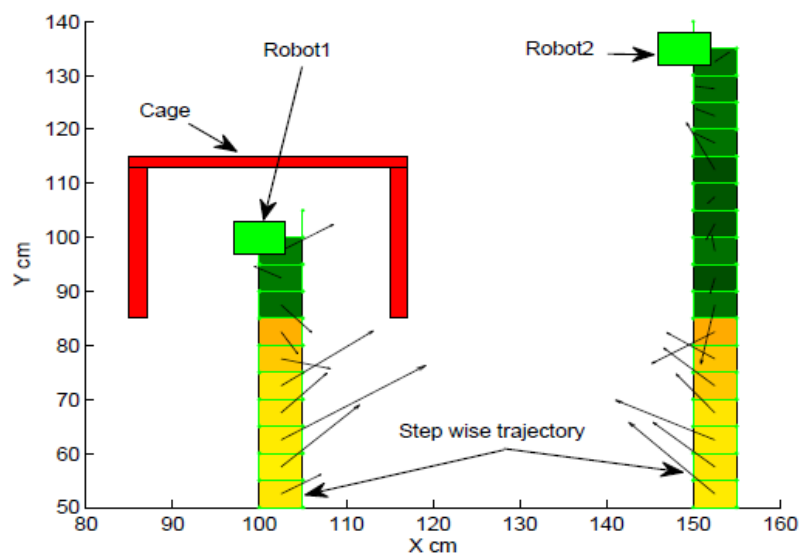

Fig. 17. One Robot is trapped in the Cage, the other Moves in a Stepwise Trajectory. 


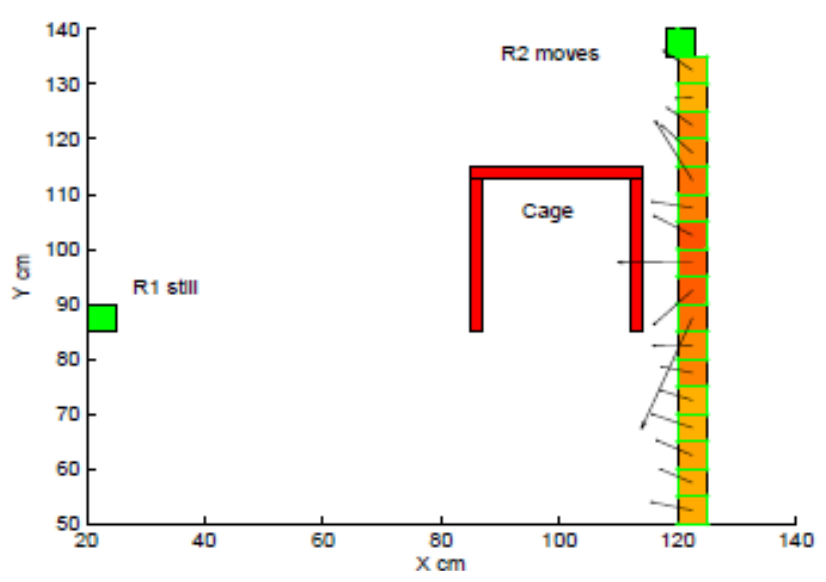

Fig. 18. One Robot Moves in a Stepwise Trajectory, and the other Stays Still.

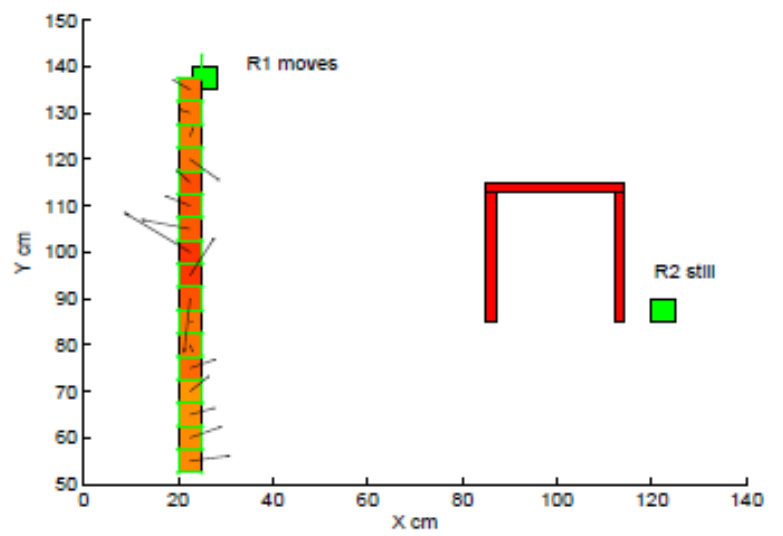

Fig. 19. One Robot Moves in a Stepwise Trajectory, and the other is Close to the Obstacle.

The scenario of Fig. 20 illustrates an obstacle and two robots in the simulation field. It shows different trajectories for one robot moving straight and the other through a stepwise course. The gradient is scattered when no LOS exists and does not contain useful information due to obstacle shadow. However, the gradient improved as the LOS became clear. The gradient is computed according to Section V's equations (5) and (7). The gradient helps find the right direction of the other robot and preserve connectivity.

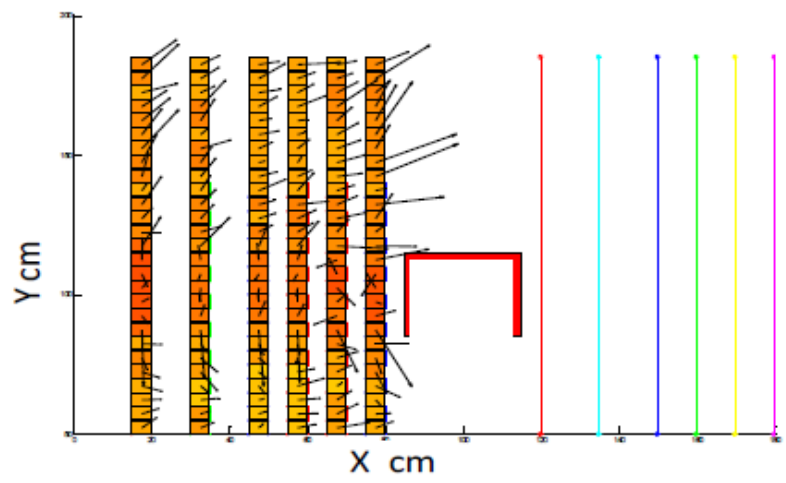

Fig. 20. Different Robots Trajectory around the Cage.

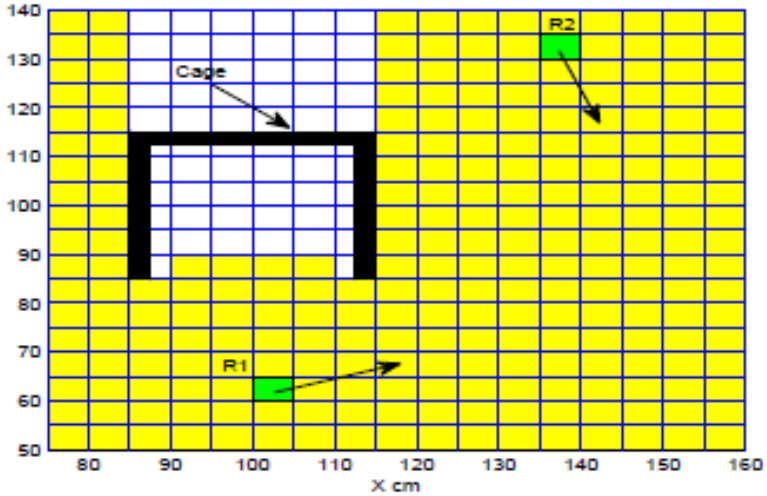

Fig. 21. Configuration Space of Two Robots.

\section{THE EXPERIMENTAL RESULTS OF THE GRADIENT ALGORITHM}

Section III explains that the experimental robot field is divided into equal grids. Then, two robots move, measure SS and compute the gradient for any two grids in the area marked yellow in Fig. 21. Next, we created a database containing the robot's position, measured SS, and calculated the gradient for any two grids (robot's location) at time t. The main steps of the algorithm are illustrated in Fig. 22. For example, at time $t=0$, two robots are placed at $x_{0}^{(1)}=20 ; y_{0}^{(1)}=5$ and robot 2 starts at $x_{0}^{(2)}=40 ; y_{0}^{(2)}=5$ in the field. Fig. 23 depicts the trajectories of the robots resulting from running the gradient algorithm, and results confirm that the algorithm helps evade obstacles' shadows and preserve communications. Fig. 24 depicts another scenario where the robots are placed in front of the obstacle at $\left(x_{0}^{(1)}=18 ; y_{0}^{(1)}=14\right)$ and the other robot at $\left(x_{0}^{(2)}=34 ; y_{0}^{(2)}=16\right)$. The gradient algorithm exhibits promising results to preserve communication between mobile robots.

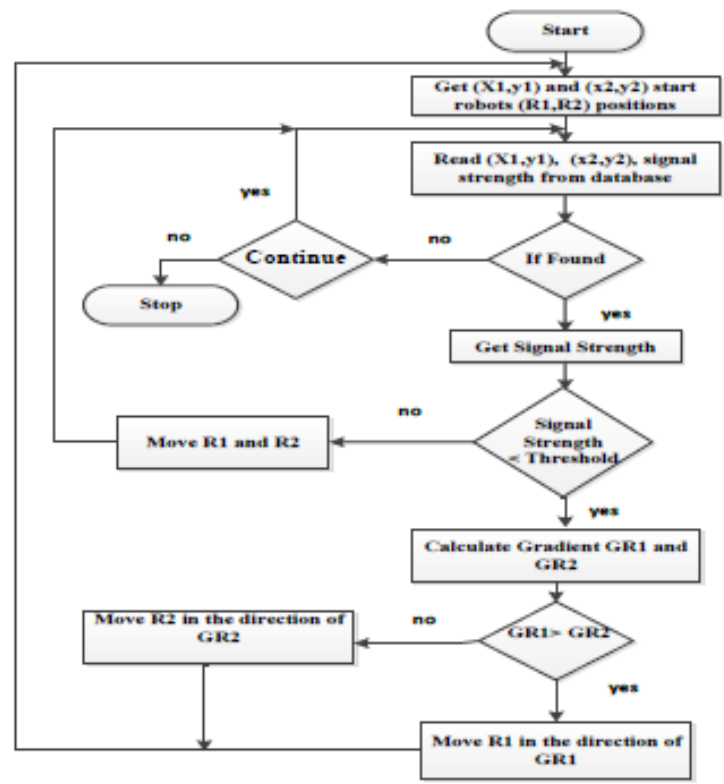

Fig. 22. Gradient Algorithm Flowchart. 


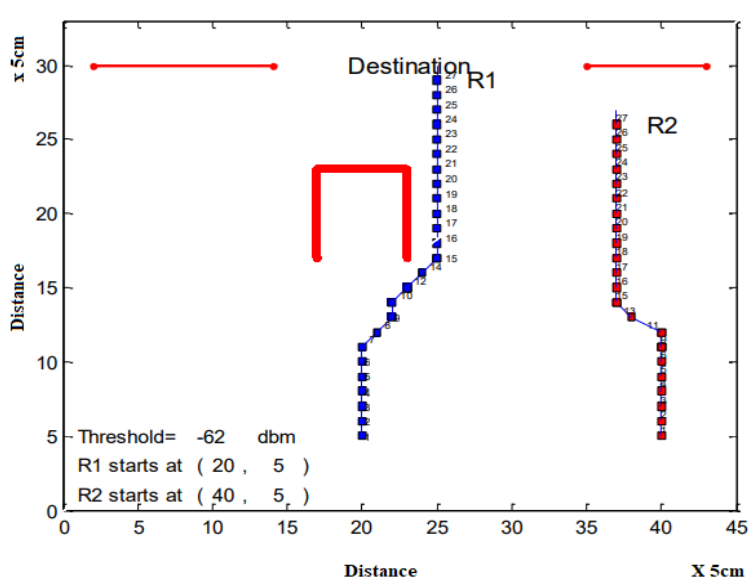

Fig. 23. Robots Start at $x_{0}^{(1)}=20 ; y_{0}^{(1)}=5$ and $x_{0}^{(2)}=40 ; y_{0}^{(2)}=5$ at Time $\mathrm{t}=0$.

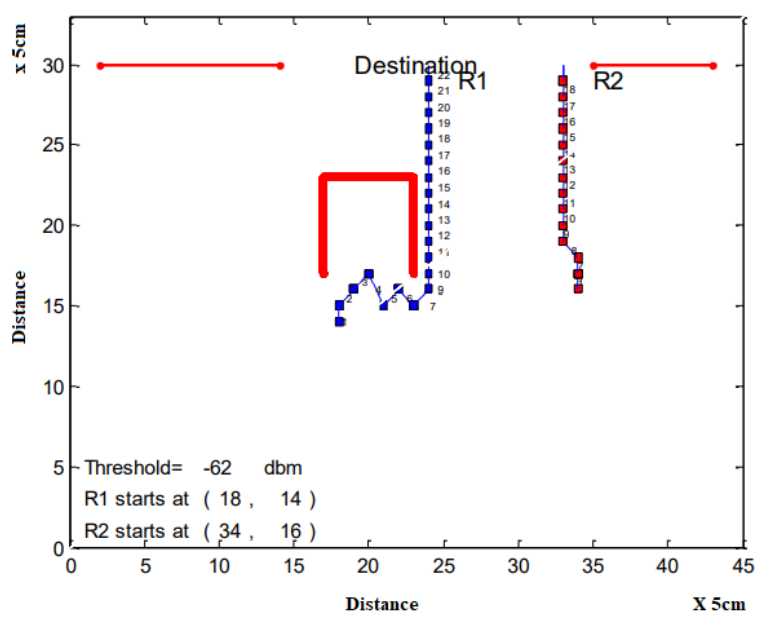

Fig. 24. Robots Start at $\left.x_{0}^{(1)}\right)=18 ; y_{0}^{(1)}=14$ and $x_{0}^{(2)}=34 ; y_{0}^{(2)}=16$ at time $\mathrm{t}=0$.

Another scenario is depicted in Fig. 25, where one robot faces the obstacle at $x_{0}^{(1)}=17 ; y_{0}^{(1)}=5$ and the other at $x_{0}^{(2)}=$ $38 ; y_{0}^{(2)}=5$ at time $t=0$. The results confirm the algorithm's success in preserving communication.

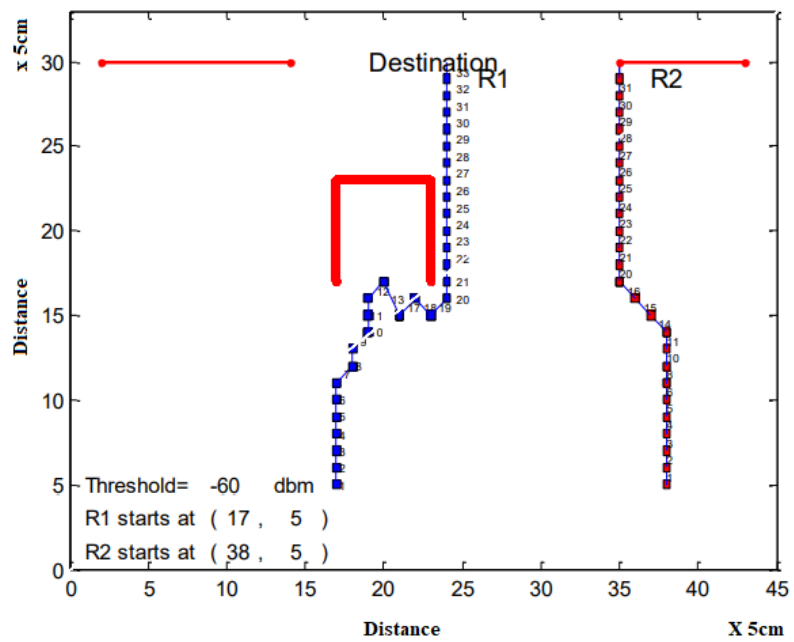

Fig. 25. Robots Start at $\left.x_{0}^{(1)}\right)=17 ; y_{0}^{(1)}=5$ and $x_{0}^{(2)}=38 ; y_{0}^{(2)}=5$ at time $\mathrm{t}=0$.

\section{THE EXPERIMENTAL RESULTS OF THE PMCA}

The HMM results of the RFMR method demonstrate the detection of obstacles on the robotic path and determine the type of distance from the robot path and the size of the approximate obstacle. PMCA uses HMM results to encourage the robot to continue moving through the current trajectory based on the length of the segments covered by the robot. If the segment's length is equal to half or greater than the estimated obstacle size, then the robot continues forward. Otherwise, the robot stops and returns to a position with robust signal strength, as shown in the scene in Fig. 26. Afterward, the robot runs a gradient algorithm to determine the strong SS direction. After that the robot moves in the gradient trend and re-establishes connectivity, as shown in the scene in Fig. 27. Algorithm 1 and Fig. 15 illustrate the PMCA mechanism.

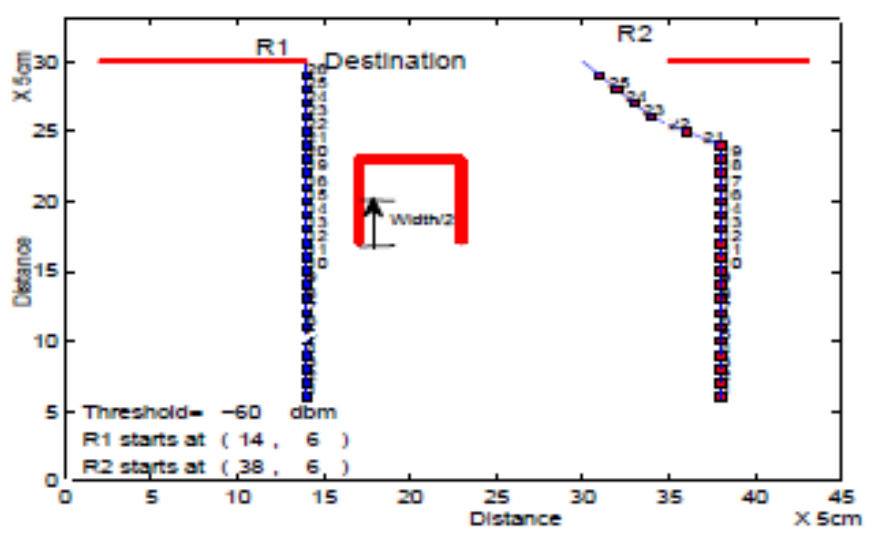

Fig. 26. PCMA uses Three Observations.

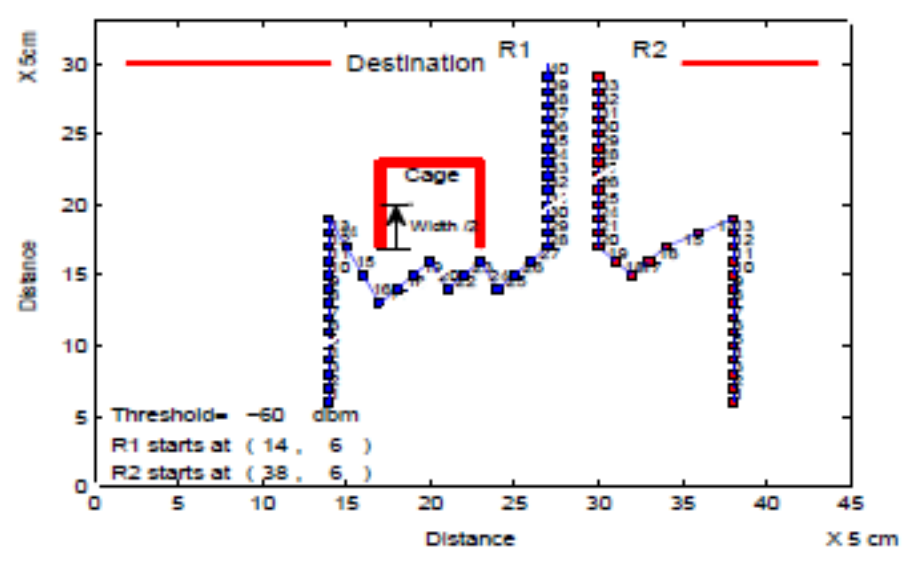

Fig. 27. PCMA uses Two Segments.

\section{CONCLUSION}

The article introduces Radio Frequency Mapping Environment Recognition (RFMR), gradient, and proactive robot motion control algorithms (PMCA). Thus, we conducted many simulations and physical experiments to assess the proposed method's performance. Consequently, this work presents promising solutions and becomes a competitive alternative for the routing and maintaining broken links problems in robot networks. Furthermore, extensive 
simulation and physical experiments will be conducted to validate the recognition of different RF obstacles. Also, obstacle parameterization and generalization approaches will be addressed in future studies.

\section{REFERENCES}

[1] Y. Wu , X. Ren, H. Zhou, Y. Wang. and X. Yi. "A Survey on MultiRobot Coordination in Electromagnetic Adversarial Environment: Challenges and Techniques," in IEEE Access, 2020, vol. 8, pp. 5348453497.

[2] M. Lindhe, H. Johansson, A. Bicchi. "An experimental study of exploiting multipath fading for robot communication," in Proceeding of Robotics: Science and Systems,2007, Atlanta.

[3] R. M. Voyles, J. Bae, A. Larson. and M. Ayad. "Wireless video sensor network for sparse, resource-constrained, multi-robot teams," in Journal of Intelligent Service Robots,2009.vol. 2, no. 4, pp.235--246.

[4] J. Bae. and R. M. Voyles. "Wireless video sensor network from a team of urban search and rescue robots," in International Conference on Wireless Networks, 2006.

[5] N. Michael, M. M. Zavlanos, V. Kumar and G. J.Pappas." Maintaining connectivity in mobile robot networks," in International Symposium on Experimental Robotics. July 2009, Athena, Greece.

[6] D. Hahnel, B. Ferris. and D. Fox. "Gaussian processes for signal strength-based location estimation," in Proceeding of Robotics: Science and Systems, 2006, Philadelphia, PA.

[7] V. Loscri, E. Natalizio. and C. Costanzo. "Simulations of the impact of controlled mobility for routing protocols," in EURASIP Journal on Wireless Communications and Networking, July 2010.

[8] A. Purohit, S. Zheng, F. Mokaya, and P. Zhang." Sensorfly: Controlledmobile sensing platform for indoor emergency response applications," in Information Processing in Sensor Networks (IPSN), 10th International Conference, 2011.

[9] A. Ghaffarkhah, and Y. Mostofi. "Path Planning for Networked Robotic Surveillance," in Signal Processing, IEEE Transactions, July 2012, vol. 60, no. 7, pp. 3560-3575.

[10] T. Kanungo, M. David., S. Nathan, Netanyahu, D. Christine., S. Ruth, and Y. Angela. "An efficient k-means clustering algorithm: Analysis and implementation," in IEEE Transactions on Pattern Analysis and Machine Intelligence, July 2002, vol. 24, no. 7, pp. 881--892.

[11] L. R. Rabiner. "A tutorial on hidden Markov models and selected applications in speech recognition, "Feb 1989, vol. 77, pp. 257 --286.

[12] He. Zhihai and Wu. Dapeng. "Resource allocation and performance analysis of wireless video sensors," in IEEE Transactions on Circuits and Systems for Video Technology, May 2006,vol. 16.

[13] M. Ayad, and R.Voyles. "Physical Link Maintenance and Logical Message Routing Integration for Robotic Network Connectivity," in Vehicular Technology Conference, 2019, IEEE. Hawaii.

[14] J. Sweeney, T. J. Brunette, Y. Yang, and R. Grupen. "Coordinated teams of reactive mobile platforms'" in International. Conference on Robotics and Automation (ICRA). IEEE, 2002, vol. 1, pp. 299--304.

[15] J. Fink, and V. Kumar. "Online methods for radio signal mapping with mobile robots network," in International Conference on Robotics and Automation(ICRA, IEEE, 2010.

[16] J. N. Twigg, J. R. Fink, P. L. Yu, and B. M. Sadler. "RSS gradientassisted frontier exploration and radio source localization," in International. Conference on Robotics and Automation(ICRA). IEEE, May 2012, pp. 889--895.

[17] Yi. Sun, X. Jizhong, Li. Xiaohai, and F. Cabrera-Mora. "Adaptive source localization by a mobile robot using signal power a gradient in sensor networks," in IEEE Global Telecommunications Conference, 2008, pp. 1 --5.

[18] Yan. Yuan, and Y. Mostofi. "Co-optimization of communication and motion planning of a robotic operation in fading environments," in Signals, Systems, and Computers (ASILOMAR) Conference Record of the Forty Fifth Asilomar Conference, Nov. 2011, pp. 1455--1460.

[19] J. Fink, V. Kumar, N. Michael, and A. Kushleyev." Experimental characterization of radio signal propagation in indoor environments with application to estimation and control," in IEEE International Conference on Intelligent Robots and Systems, 2009.

[20] M. Ani Hsieh, C., Anthony , V. Kumar, and J. Camillo. "Maintaining network connectivity and performance in robot teams," Research articles. Journal Field Robot, (2008).

[21] M. M. Zavlanos,. and G. J. Pappas. "Distributed connectivity control of mobile networks," in IEEE Transactions on Robotics, Dec. 2008, vol. 24, no. 6, pp. $1416--1428$.

[22] Ji. Meng, and M. Egerstedt." Distributed coordination control of multiagent systems while preserving connectedness," in IEEE Transactions on Robotics, Aug. 2007, vol. 23, no. 4, pp. 693 --703.

[23] M. A. Hsieh, A. Cowley, V. Kumar, and CJ. Taylor." Towards the deployment of a mobile robot network with end-to-end performance guarantees," in Proceedings IEEE International Conference on Robotics and Automation (ICRA), May 2006, pp. 2085 --2090.

[24] F. Zeiger, N. Kraemer, and K. Schilling. "Commanding mobile robots via ad-hoc wireless networks - a comparison of four ad-hoc routing protocol implementations," in IEEE International Conference on Robotics and Automation (ICRA).

[25] Y. Mostofi, M. Malmirchegini, and A. Ghaffarkhah.” Estimation of communication signal strength in robotic networks," in IEEE International Conference on Robotics and Automation (ICRA), May 2010, pp. $1946--1951$.

[26] D. P. Spanos and R. M. Murray. "Robust connectivity of networked vehicles'" in IEEE Conference on Decision and Control Conference, Dec. 2004, vol. 3, pp. 2893 -- 2898 Vol.3.

[27] M. Ayad, and, Mo. Ayad, " Investigates the Radio Frequency Obstacle Effects on Wireless Signal for Robot Communication, "in Future Technologies Conference, FTC 2021, Oct. 2021.

[28] M. Ayad, and R. Voyles, "R.F. Mapping for Sensor Nodes Connectivity and Communication Signal Recovery," Computing Conference 2021, London, United Kingdom, July 2021.

[29] CST2021, CST Studio Suite 3D EM simulation and analysis software.

[30] M. Ayad, and R. Voyles, "Message Routing and Link Maintenance Integration for Robotic Network Connectivity," in Future Technology Conference (FTC- 2020), Vancouver, Canada, Nov. 2020.

[31] M. Ayad, J. J. Zhang, R. Voyles, M. H. Mahoor. "Mobile robot connectivity maintenance based on RF mapping," in IEEE/RSJ International Conference on Intelligent Robots and Systems (IROS). 2013.

[32] D.Jurafsky. and J. H. Martin. Speech and language processing,,2000, in Prentice Hall, Inc.

[33] Maya Nayak and Bhawani Sankar Panigrahi ." Advanced Signal Processing Techniques for Feature Extraction in Data Mining," in International Journal of Computer Applications, April 2011, vol. 19, no. 9, pp. 30-37.

[34] M. Ayd, J. J. Zhang, R. Voyles and M. H. Mahoor.” Electromagnetic Field Recognition for Proactive Robot Communication Connectivity Maintenance," 2012 Asilomar Conference on Signal, Systems, and Computers (Asilomar), 2012.

[35] M. A. Ayad," Meta-Routing: Synergistic Merging of Message Routing and Link maintenance," at https://digitalcommons.du. edu/etd/40. 